\title{
SOME APPROACHES TO THE ROLLING WHEELS' DYNAMICS MODELLING IN THE WEIGHT-IN-MOTION PROBLEM
}

\author{
Alexander Grakovski, Yuri Krasnitski, Igor Kabashkin, Yuri Sikerzhitski \\ Victor Truhachov
}

\author{
Transport and Telecommunication Institute \\ Lomonosova 1, Riga, LV 1019, Latvia \\ Ph.: (+371)67100654.Fax: (+371)67100535.E-mail:avg@tsi.lv
}

\begin{abstract}
Some possibilities of fibre-optic sensors (FOS) application for measuring the weight of moving vehicles realized in weightin-motion (WIM) systems are discussed. As the first, the model of small-buried seismic sensor transient response excited by a car tyre interaction with asphalt-concrete road pavement is proposed. It is supposed that a seismic wave received by the sensor is the vertical component of surface Raleigh wave. The model is based on supposition that a tyre footprint is acceptable to consider as some array of point sources of these waves. The proper algorithms permit to vary different parameters of the array excitation, as to footprint dimensions, load distribution, car velocities and others. The set of Matlab codes is worked out for seismic pulses modelling and processing.

The second way considered is to simulate the FOS signal in the basis of differential equations describing a deformable
\end{abstract} wheel behaviour, or wheel oscillations, in order to identify relations with optoelectronic mechanical parameters. An attempt to find the mass of the vehicle is based on minimizing the discrepancy between the actual FOS signal and the solution of the differential equation. The accuracy of the evaluated weight depends on many external factors, the mathematical modelling of them are expressed in the numerical values of the coefficients and external stimuli. The influence of these factors are analysed and tested by simulations and field experiments.

One of ideas in dynamic weighing problem solution should consist in evaluation of position of virtual gravity centre of the vehicle in time. The processing algorithm of the data received from the FOS is proposed based on conception of database retaining in some reference system memory. Certain requirements concerning the elements and blocks of the algorithm are defined as well. The reference system is realized as the digital filter with the finite impulse response. The method to estimate the filter coefficients is worked out. Several experiments with this algorithm have been carried out for the vehicle identification with the reference loads adopted from real data.

The different factors have an influence on the measurement accuracy of FOS. The roadbed features, temperature, nonlinearities and delay effects in FOS are among them. The results of laboratory and field measurements with FOS responses to different axle's loadings are presented. Charging and inertial characteristics of FOS under the impact of various external factors (protective cover, temperature, contact area, and installation mode especially) as well as their approximations are investigated. It is found that the final calibration of the FOS has to be done individually and only after it has been installed in the pavement. Certain methods and algorithms of linearization, as well temperature and dynamic errors compensation of FOS data are discussed.

Keywords: Weight-in-motion, WIM systems, fibre optic sensors, sensor's sensitivity, tyre footprint, road pavement reaction, sensor response modelling

\section{Introduction}

Economic situations in majority countries are visibly affected by range of automotive load transportation, and, therefore, by satisfactory condition of road networks. Degree of road deterioration is determined mainly by traffic activity and excessive brutto weight of laden trucks. In accordance with present standards, certain loading for a single axle of a motor car has to be restricted to some maximum allowable value. Exceeding of it assumes proper sanctions. Sensor networks for weighing road vehicles are the tools for the problems of maintaining the quality of road surfaces solution in conditions of growth of traffic intensity in the European Union countries and creation of the traffic control intelligent transport systems. It is supposed that the modern WIM system should to be ensuring the measurement errors of $2-5 \%$ from true axle loading on the truck speed of 50-100 km/h regardless of the sensor type.

Very promising way to estimate the loading on certain axle is application of WIM systems [1-6]. There are many versions of WIM. Some from them have used sub-surfaced small-buried seismic sensors within road pavement [3, 4]. Nowadays, the use of fibre-optic sensors, based on changing of the parameters of the optical signal from optical fibre strain under the weight of passing transport [1], takes an exclusive popularity in the problems of transport telematics. These sensors are relatively durable and inexpensive to manufacturing and operating. However, due to the low accuracy of measurement of weight (especially in a moving object case), and high dependence on weather conditions, they are used mainly as a vehicle's motion detectors only. 
Output response of that sort of sensor is a time series of some non-stationary transient signals. The number of pulses is equal to the number of axles of the motor car which is passed over the sensor at that time interval. At first sight all the pulses appear to be similar one another. However, some theoretical considerations, as well analysis of experimental data, demonstrate certain differences existing among them. For successful designing a WIM system the nature of these delicate differences ought to be discovered and interpreted.

Thus, the goal of a WIM system modelling is to work out a method to filter individual differences in features of these pulses. It is natural that problems related to internal/external factors influenced on FOS transient forms ought to be studied too. That is the range of questions supported in this paper.

The organization of the paper is as follows. Some qualitative considerations concerning wheelroad interaction and theoretical modelling of some possible FOS input forms are studied in Section 2. The problem relates to calculating of a basic seismic pulse excited by a unit point mass body moving with some friction along a horizontal road. The model of that pulse excited by tyre-road contact footprint is suggested. The tyre footprint is considered to be some discrete dynamic array of surface Raleigh waves sources with exciting loadings distributed all along the footprint. If a sensor depth position (that is a distance of it from pavement air surface) and tyre footprint dimensions are assigned as some initial conditions the proposed model permits to a) vary the function which describes tyre contact pressure distribution inside the footprint contour, b) specify an automobile velocity, c) match a rolling road resistance coefficient, d) take into account of wind velocity component oriented along the road, etc. Results of modelling are sensor response forms which ought to comply with conditions specified above. These forms may be used as a starting material for formulation of the target inverse problem namely estimation of loads on individual axles of a vehicle passed over the WIM sensor. Some potentials of that model are demonstrated with examples.

The aim of the Section 3 is in describing the approach to simulating the signal of FOS based on differential equations reflected deformable wheel characteristics [12-16] and identification of relations with optoelectronic and mechanical parameters of a sensor. Finding the mass of the vehicle is decided by minimizing certain discrepancy between the actual FOS signal and the solution of the differential equation of wheel oscillations named above. Mathematical modelling of external perturbed factors [16] is expressed in the numerical values of the coefficients and external forcing stimuli. The impact of these factors on the accuracy of WIM systems were analyzed and tested by simulations and field experiments.

The Section 4 is devoted to study an adaptive method for system identification in order to determine the type and weight of the vehicle. This method consists in using a database of signals obtained with the fibre optic sensor when a truck of the certain weight driving on it.

Signal data from the output of fibre optic sensor based on comparison of the distribution center of mass along the axes of the vehicle in the static and dynamics are reduced to a single scale on the time axis. This procedure uses data on vehicle speed.

In its turn, comparison of signal output from the fibre optic sensor for dynamic and static weighing using adaptive filtering techniques, allows us to create a database of equivalent reference systems in the form of a set of stationary filters for trucks with the certain weight.

After processing the signals using the proposed method by comparing the response at the output of a stationary filter, simulating the operation of the master system and the response to the output fibre-optic sensor, after the passage of the vehicle of the unknown type, weight of the truck is estimated.

In Section 5 extensive studies is carried out of discrepancies in pulse forms registries from FOS as the results of laboratory and field natural experiments under different initial conditions (i.e., truck loads and velocities, as the weather too). The results of the laboratory and field measurements are presented, as well as the load and inertial characteristics and their approximations, obtained for fibre-optic sensor under the impact of various external factors (protective cover, temperature, contact area, and especially installation). We discuss the algorithms for linearization, calibration and methods of temperature compensation of fibre-optic sensors when using them in weight in motion (WIM) applications.

Last trends on WIM issues indicate that FOS sensors are based on two main principles: Bragg grating (the change of diffraction in a channel under deformations) [17] and change under deformations of the fibre optical properties (transparency, frequency, phase, polarization) [27]. The change of transparency (the intensity of the light signal) of SENSORLINE experimental sensors [28], as basic operating principle, is considered in this study.

Most popular are the multi-sensor systems (MS-WIM) [29], where the accuracy of measurements is obtained by statistical processing of 6-20 or more sensors, situated in sequence into the pavement at some distance to each other. Obtaining the measurement errors of $<5 \%$ this approach takes the expenses, 
compatible with the bending plates and needs the reconstruction of roadside surface. The aim of the present study is to obtain the measurement errors of $2-10 \%$ without multiplying of sensors.

This work was worked out by supporting of ERDF funding project "Fibre Optic Sensor Applications for Automatic Measurement of the Weight of Vehicles in Motion: Research and Development (2010-2012)".

\section{Transient Response of a Small-Buried Seismic Sensor}

Output response of that sort of sensor is a time series of some non-stationary transient signals. The number of pulses is equal to the number of axles of the motor car which is passed over the sensor at that time interval. At first sight all the pulses appear to be similar one another. However, analysis of experimental data, demonstrate certain differences existing among them. For successful designing a WIM system the nature of these delicate differences ought to be discovered and interpreted.

The goal of a WIM system designing is to work out a method to filter individual differences in features of that pulses. In this section the algorithm is developed based on modelling of a small-buried seismic sensor response excited by forced impact distributed along a car tyre footprint to asphalt-concrete road pavement. It is supposed that a seismic wave perceived by the sensor is the vertical component of surface Raleigh wave [7,8] propagating in the road pavement top layer.

It is proposed the tyre footprint should to be consider as some discrete array of point sources of surface Raleigh waves each with own exciting loading distributed along the footprint. A transient signal created by interferences of these waves is received by the seismic sensor.

\subsection{Some Features of Wheel - Road Interaction}

A qualitative picture of wheel - road interaction (Figure 1) is set out in [9]. This interaction takes place within the bounds of some contact pavement surface referred to as a tire footprint.

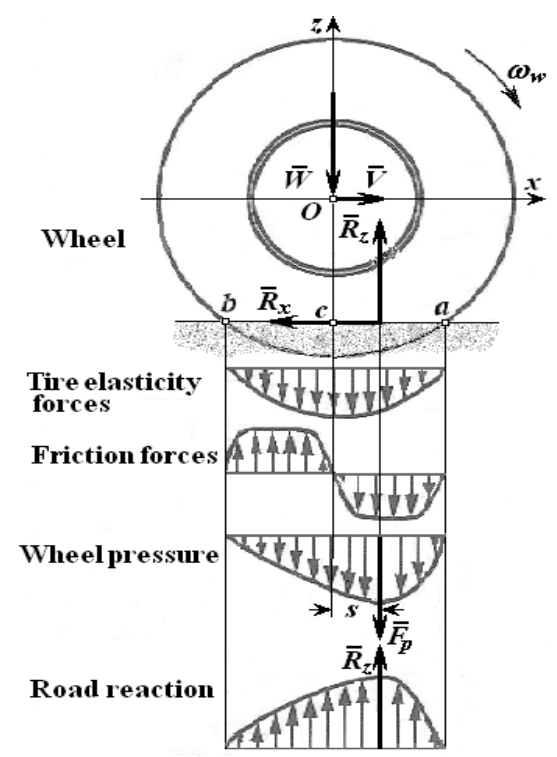

Figure 1. Wheel - road interaction picture (adapted from [9])

An axle loading $\boldsymbol{W}$ is translated to a footprint as a non-uniformly distributed wheel pressure. It is described by a certain function $\boldsymbol{F}_{p}(\boldsymbol{x}, \boldsymbol{y})$, which is depended on tire elasticity forces depicted by even temporal modes, as well as friction forces caused by elements of a pneumatic tire tread. As the wheel is in rolling motion along the axis $\mathrm{x}$ with some linear velocity $\boldsymbol{V}$ and angular velocity $\omega_{\mathbf{w}}$, the radial deformation of the tire tread is changed abruptly in time with a really complicated manner. It is amplified in the tread front part, but it is reduced in the back part of it. These reasons are reflected in the friction forces as the dominated odd modes.

As a result, at the $a-c$ part of footprint the friction forces have the same directions as the tire elasticity forces, but along the $c-b$ part their directions are opposed. Therefore, wheel pressure $\boldsymbol{F}_{\boldsymbol{p}}$ and road reaction $\boldsymbol{R}_{\boldsymbol{z}}$ diagrams are non-symmetrical relative to the vertical axe $z$ passed through the wheel centre.

The extreme of pressure and reaction forces are displaced from the axe $z$ by the shift $s$. Thus, a value of $s$ should serve as a measure of wheel - road interaction. Unfortunately, exclusive multifactor complexity of considered process not yet permits to create the mathematically strict description of it.

\subsection{Response on a Unit Point Mass Movement}

The problem described in this part is derivation of a seismic pulse form excited by a unit point mass body moving with certain friction along a smooth horizontal road. Figure 2 shows the sketch of the task. An omni-directional (isotropic) seismic sensor is placed on some depth $\mathrm{h}$ from road surface and it is superposed with the origin $O$ of Cartesian coordinates system. A point body moves from initial position $\boldsymbol{t}=\boldsymbol{0}, \boldsymbol{x}=\boldsymbol{x}_{\boldsymbol{0}}$ to right along the plain pavement surface with constant velocity $\boldsymbol{V}$. It is supposed that $\boldsymbol{x}_{\boldsymbol{0}}$ is a negative value. The body experiences an influence both force of weight $\boldsymbol{W}$ and friction force $\boldsymbol{F}$ too. 


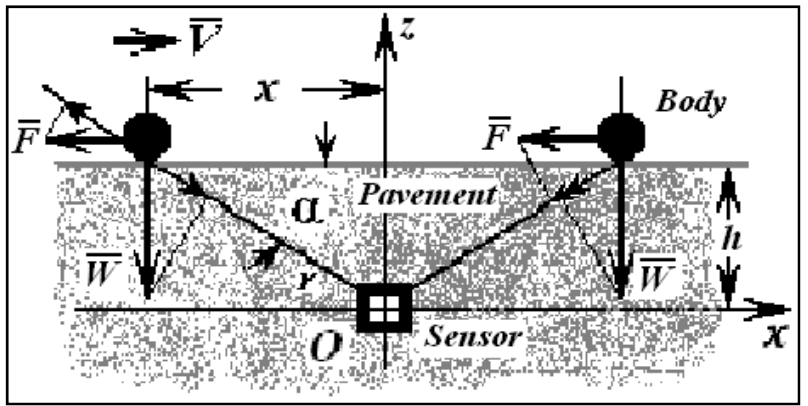

Figure 2. Excitation of seismic sensor by a unit point mass body movement

Instantaneous position of the body concerning the sensor describes by distances $x, r$ and an angle $\alpha$ where

$$
\boldsymbol{x}=\boldsymbol{x}_{0}+\boldsymbol{V t}, \quad \boldsymbol{r}=\left(\boldsymbol{x}^{2}+\boldsymbol{h}^{2}\right)^{1 / 2}, \quad \sin \alpha=\boldsymbol{h} / \boldsymbol{r}, \quad \cos \alpha=\boldsymbol{x} / \boldsymbol{r}
$$

Movement of this body excites seismic oscillations in pavement layer propagating along the road surface as the Raleigh type waves with velocity $\boldsymbol{V}_{\mathbf{R}}$. The current pressure $\boldsymbol{P}$ perceived by the sensor depends on the instantaneous sum of projections onto running radius - vector $\boldsymbol{r}$ of the forces $\boldsymbol{W}$ and $\boldsymbol{F}$.

It is seen from Figure 1 that projections of $\boldsymbol{W}$ would be alter their directions as soon as the sign of $x$ is changed, hence, one can describe

$$
P=(W \cdot \sin \alpha-F \cdot \cos \alpha) / \sqrt{r},
$$

where it has been taken into account the reverse squared root dependence of Raleigh surface wave intensity from distance $[4,5]$. If a sensor has to response on normal, or $z$, component of force $\boldsymbol{P}$ only, then it is necessary to project the force in Eq. (2) to $z$-axis. Allowed for (1), the result can be written as

$$
\boldsymbol{P}_{z}=\boldsymbol{W}\left(1+\boldsymbol{k}_{\boldsymbol{F}} \boldsymbol{x} / \boldsymbol{h}\right) \boldsymbol{h}^{2} / \boldsymbol{r}^{5 / 2},
$$

where the value of $\boldsymbol{k}_{\boldsymbol{F}}=\boldsymbol{F} / \boldsymbol{P}$ may be considered as a rolling friction coefficient.

As the Raleigh surface wave propagates along the $r$ from the point of instantaneous position of the moving body to the sensor it should be delayed in time on $\boldsymbol{t}_{\mathbf{R}}=\boldsymbol{r} / \boldsymbol{V}_{\mathbf{R}}$. Temporal scale of sensor has to take it into account. Therefore, "sensor time" $\boldsymbol{t}_{\mathrm{s}}$ has to look as

$$
t_{s}=\left(x-x_{0}\right) / V+\left(r-r_{0}\right) / V_{R},
$$

where $\boldsymbol{r}_{\mathbf{0}}=\left(\boldsymbol{x}_{\mathbf{0}}{ }^{2}+\boldsymbol{h}^{2}\right)^{\mathbf{1} / 2}$. Equations (3) and (4) may serve as a basis for modelling of sensor responses time forms initiated by a moving body. Certain results are presented on Figure 3 with some variations of $\boldsymbol{k}_{\mathrm{F}}$.

It should be noted that in accordance with Eqs. (1) and (4) the distance $x$ and sensor time $t_{\mathrm{s}}$ are in nonlinear relation due to the second item in (4). It is especially significant in a region of small times.
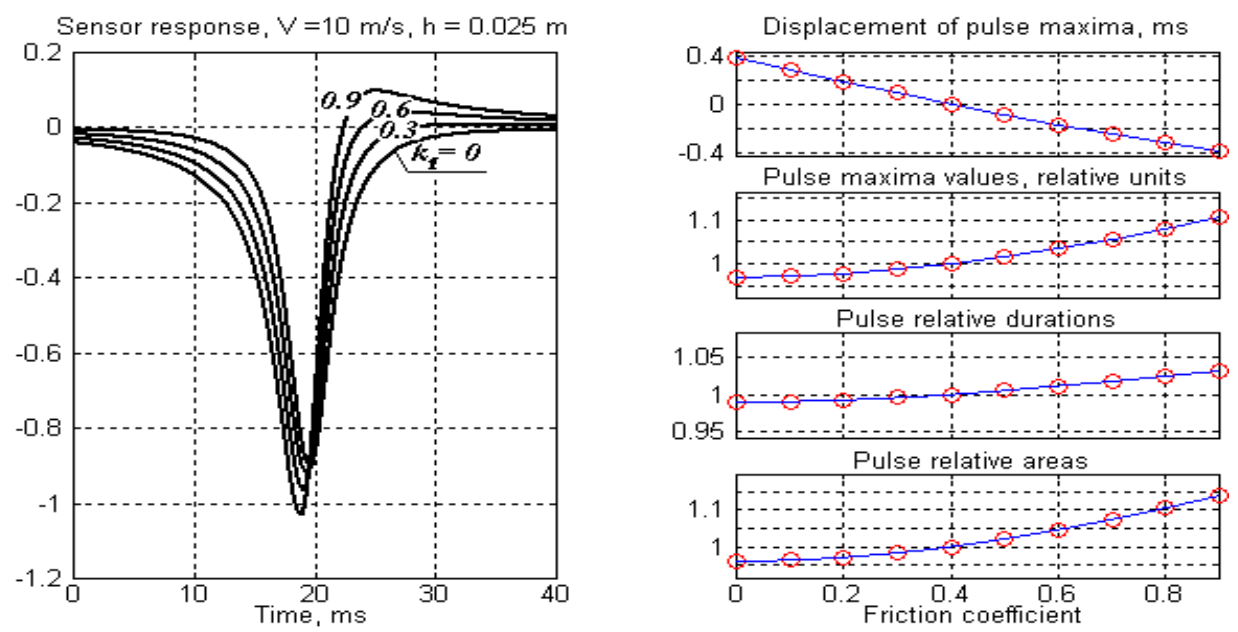

Figure 3. Pressures pulses forms versus rolling friction coefficients 
The plots on Figure 3 represent the results of seismic wave propagation in asphalt-concrete road pavement. The exact value of Raleigh wave velocity is unknown but it perhaps is about $V_{\mathbf{R}}=400 \mathrm{~m} / \mathrm{s}$ [10]. It is seen from the left part of Figure 3 that the growth of friction coefficient leads to certain asymmetry of sensor transient response with respect to maximum value of it. It is accompanied by increasing of pulse amplitudes, durations and areas. In the same time the maximum of the pulse is shifted with some lag in opposition to direction of motion. It is not contrary to [9]. Some features of the pulses are shown on graphs placed in right panel of Figure 3.

\subsection{Seismic Pulse Excited by Tyre-Road Contact Footprint}

The model described in the previous part has been permitted to derive a seismic pulse form excited by a unit point mass. Essentially, solution of this task should be considered as a certain Green's function. Hence, it may be used to find a response caused by motion of some finite-dimensional body with known distribution of mass along it. Such the solution is reduced to modelling of interaction of seismic pulses excited by different parts of the moving body taking into account the lags.

That approach may be conformable with the problem of sensor response excited by forced impact of a tyre to pavement. Unfortunately, any analytics, neither of mass distribution along a footprint, nor road reaction, are not discovered in accessible reference sources. The normalized function

$W(x)= \begin{cases}\sin \left(\pi x / 2 x_{\text {max }}\right), & x \in\left[0, x_{\text {max }}\right] \\ \cos \left[\pi\left(x-x_{\text {max }}\right) / 2\left(l-x_{\text {max }}\right)\right], & x \in\left[x_{\text {max }}, l\right]\end{cases}$

is proposed here to descript the distribution above as a piecewise smooth approximation where $l$ is the footprint length, $x$ is the current coordinate along footprint. The value of $x_{\max }$ is the position of maximum pressure point, or road reaction (see the shift $s$ on Fig. 1). This maximum is displaced from the point of footprint centre in the direction of motion of the car. The value of $s$ would be associated with friction coefficient and depends on road rolling resistance, car velocity, aero dynamical factors, wind vector etc. However, the numerical modeling practice have been demonstrated that more promised results have to take place with upgraded formulation of (5), namely

$W_{M}(x)=W^{\alpha}(x), \quad \alpha<1$.

More pertinent values of $\alpha$ about $0.3-0.5$ have been established with numerical experiments. Behavior of (6) under different $\alpha$ is illustrated in Figure 4. It is conformed to friction coefficient value nearly 0.3 . The curves on Figure 4 are not contrary to proper graphs which had been given in some reference sources (see Fig. 1) to explain nature of road reaction on a footprint contact at least in qualitative sense.

In order to calculate sensor transient response initiated by a body with finite sizes it is necessary to consider an equivalent seismic source in the form of 1- or 2-D discrete array. It consists of well-defined number of point sources each with own exciting loading. Every element of the array excites own Raleigh wave and makes some contribution into the normal pressure component influenced on a sensor.

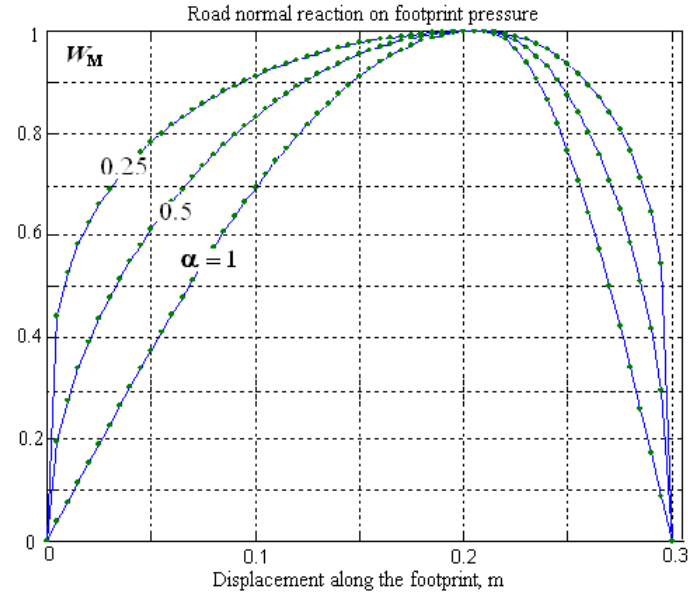

Figure 4. Normal road reaction distribution $\left(l=0.3 \mathrm{~m}, x_{\max }=0.21 \mathrm{~m}\right)$
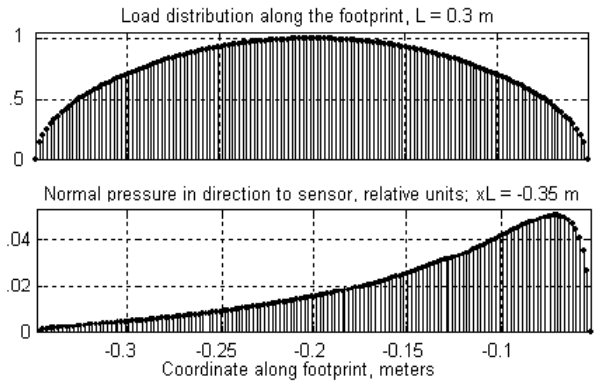

Response on footprint impact, relative units

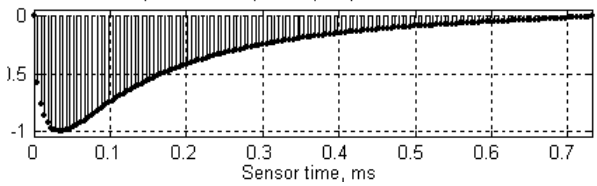

Figure 5. Pulse response from tyre footprint contact with a road 
Described approach permits to find a sensor response on short-term impact under simultaneous contact of tyre with a pavement inside footprint. The right-angled contour of the latter is supposed. Symmetric load distribution along the footprint is shown on Figure 5. The middle panel presents relative contribution into total pressure from different elements of an equivalent array.

It is supposed the sensor is situated on the depth $h=0.025 \mathrm{~m}$ from the surface. The simplest case has a place that the footprint width is supposed to be a constant. Coordinates along footprint are counted from sensor position (as the value of $x$ on Fig. 1). Finding of sensor reaction in those conditions is, in essence, a one-dimensional problem consisting in evaluating of the pulse response of the sensor with delta stimulus. Such the response after normalization is plotted on Figure 5, the lower panel.

It would be noted that natural experiment by means of the direct instrumental measurement of the pulse response is rather difficult in realization. It is possible that computer modeling is only one the way to decide this problem by relatively inexpensive tools.

\subsection{Variations of Seismic Pulse Forms}

As a function of weight, a seismic pulse form in WIM applications has to depend on many arguments and can be described by different features. Being realized as computer codes the models considered above allow analyzing relative influence of some factors on inherent structure of the pulse and picking out the most correlated with WIM aims.

In computational sense, finding of a pulse form excited by a moving array with $N$ elements reduces to calculation of some matrix. Every string in it is an elementary pulse. It is conformed to appropriately delayed motion of an individual element of the array (see Eqs. (1-4)). That matrix is

$$
\boldsymbol{P}=\left[\begin{array}{cccccccc}
\boldsymbol{P}_{11} & \boldsymbol{P}_{12} & \ldots & \boldsymbol{P}_{1 K} & 0 & 0 & \ldots & 0 \\
0 & \boldsymbol{P}_{21} & \boldsymbol{P}_{22} & \ldots & \boldsymbol{P}_{2 K} & 0 & \ldots & 0 \\
\ldots & \ldots & \ldots & \ldots & \ldots & \ldots & \ldots & \ldots \\
0 & \ldots & 0 & \boldsymbol{P}_{N I} & \ldots & \ldots & \boldsymbol{P}_{N, K-I} & \boldsymbol{P}_{N K}
\end{array}\right],
$$

where $K$ is the prescribed number of array element positions, or shifts, along $x$-axis on Figure 1. Every string in (7) is completed by zeroes in start and finish ends in order to equalize the lengths. Current position of the element $P_{\mathrm{NK}}$ determines the total length of any string. As sensor time $t_{\mathrm{s}}$ depends on $x$, nonlinearly plain addition by columns in (7) is not correct to have the right response form. Certain interpolation for every string has to be done preliminarily using the Raleigh wave minimal arrival time $h / V_{\mathrm{R}}$ as the step.

It can be observed that computation of the matrix (7) is equivalent to convolution

$$
P(x)=\int_{-\infty}^{\infty} P_{z}(x-\xi) W_{M}(\xi) d \xi
$$

of the unit point pulse (3) with the footprint loading distribution (6). The latter should be considering as a function of time which is revealed oneself in process of footprint rolling over the sensor.

As a sounding example, this method may be applied to the problem of correlation of pulse form with footprint length solved by computational experiments. In order to correspond with (7) it has been supposed the seismic signal is formed by linear combination of some delayed pulses. Each from them is excited by proper element of discrete array which is considered as a certain equivalent of the footprint. The vehicle velocity $\boldsymbol{V}$, as well as the position, or shift $s$ from a loading centre, of the road reaction $\boldsymbol{R}_{\mathrm{z}}$ maximum value (see Fig. 1), are treated as the constants. Results are shown in Figure 6. 

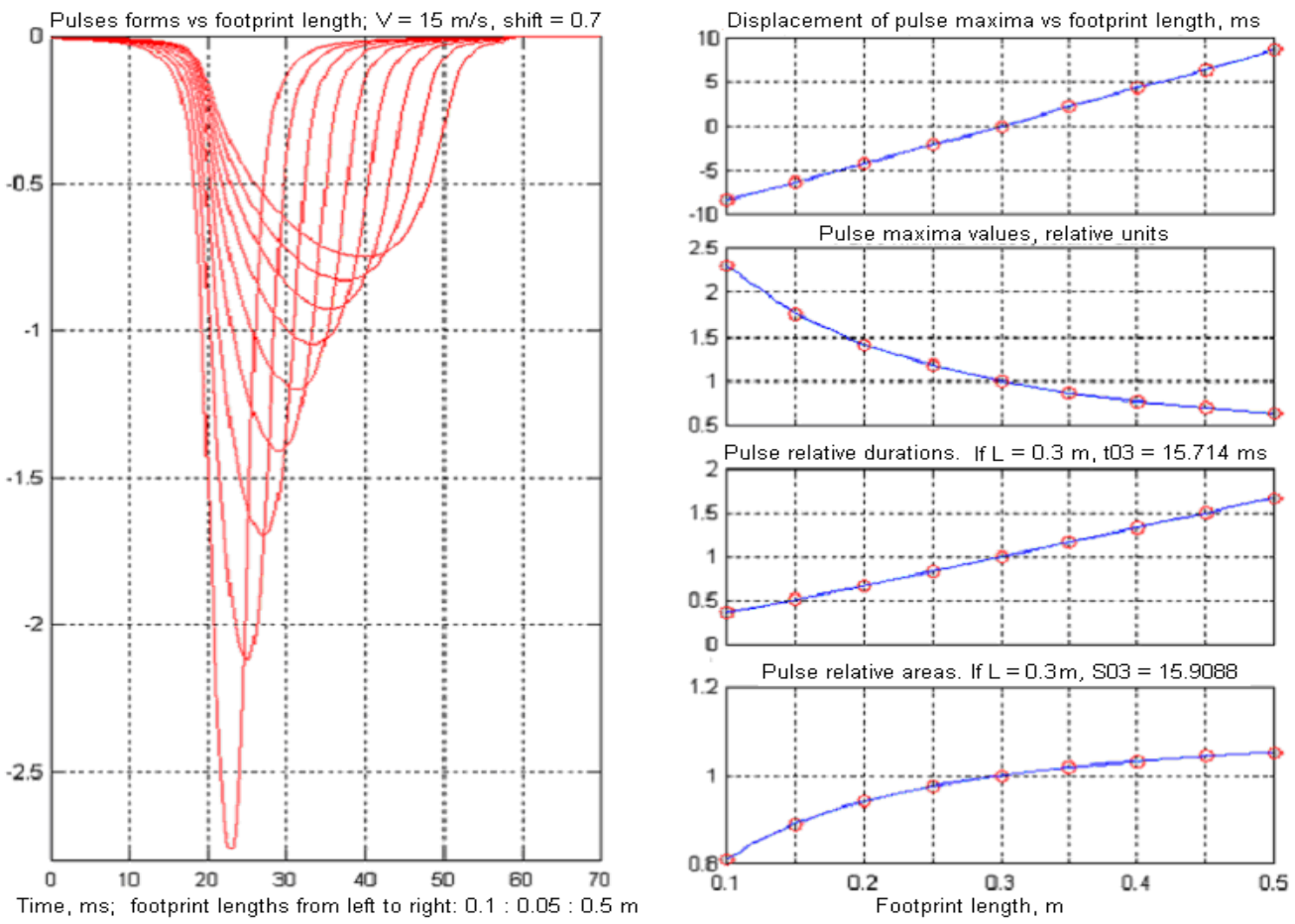

Figure 6. Forms of pulses vs footprint lengths

In particular, it is seen from the right panel of Figure 6 that durations of the pulses and displacements of the pulse maxima depend on footprint length rather linearly, in contrast to other characteristics of these transients.

The other initial conditions to modelling were used to draw the graphs on Figure 7. It is seen that variation of maximal loading shift in (6) has a predominant influence on the pulse maxima displacements only. The rest of parameters are changing just insignificantly.
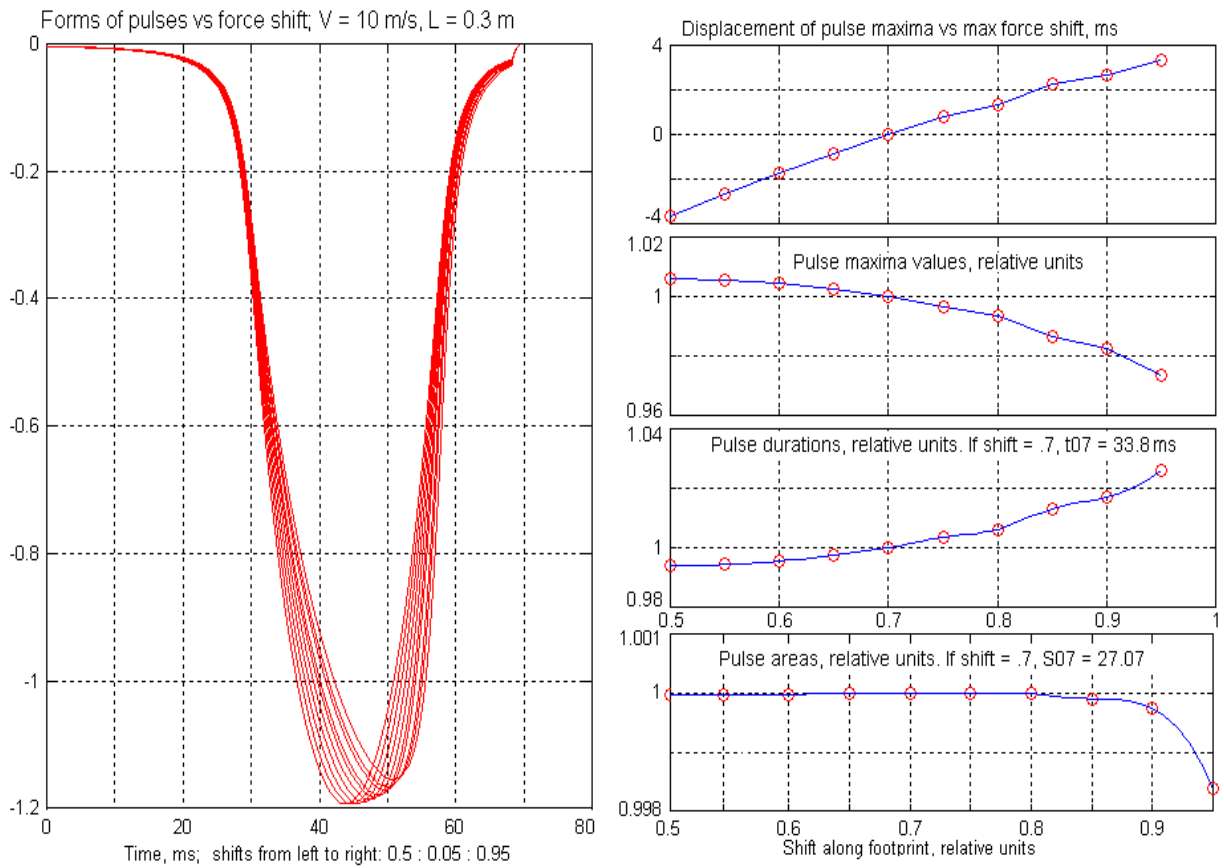

Figure 7. Forms of pulses vs maxima force shift 
Dependencies of the modelled seismic pulse parameters from car velocity are shown on Figure 8. One can observe some weak linear relation among velocity and maximal values of the pulses. The other parameters have noticeable nonlinearities with respect to this velocity.
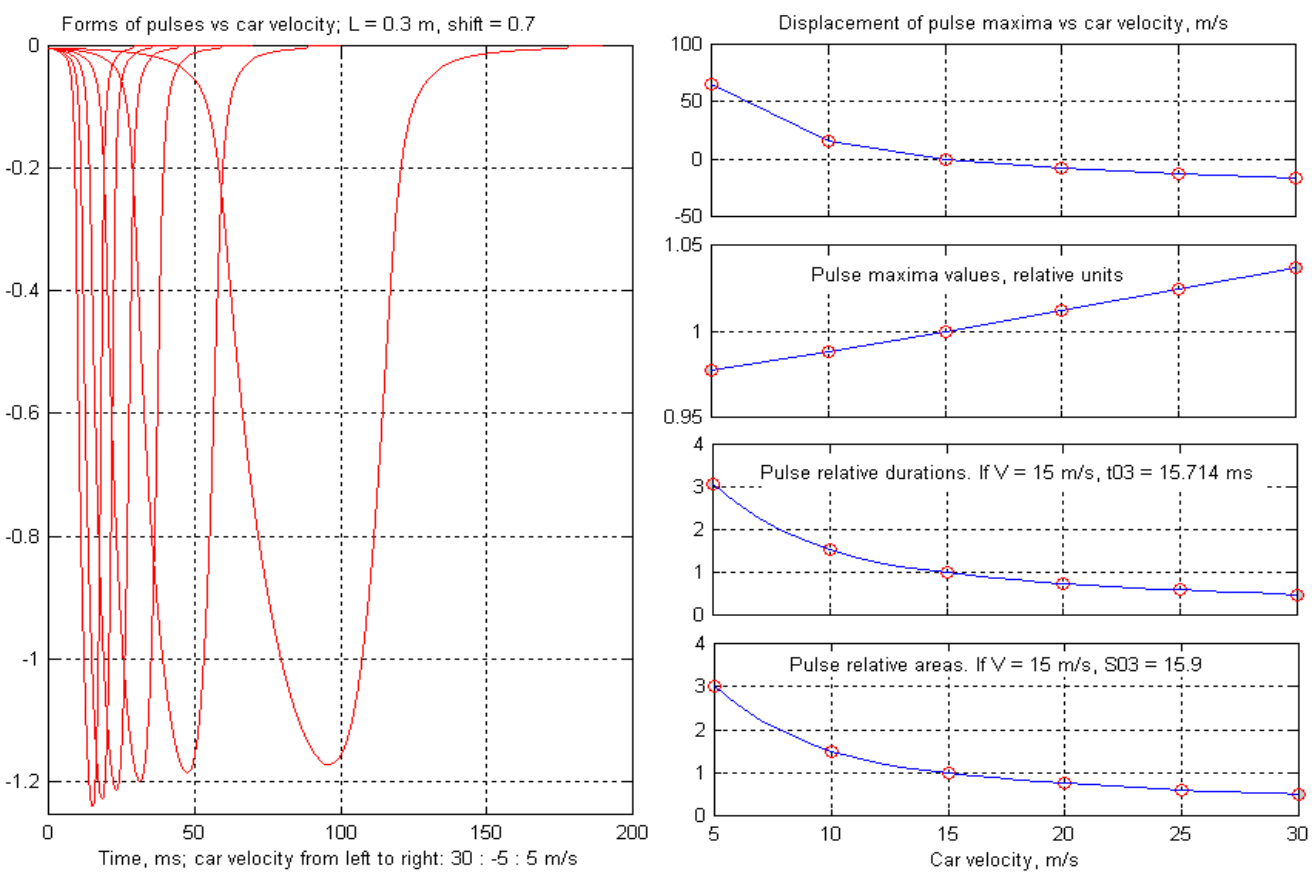

Figure 8. Forms of pulses vs car velocity

In common case, if a sensor depth position and tyre footprint contour dimensions are assigned as some initial conditions the proposed model permits to:

a) vary the function which describes tyre contact pressure distribution inside the footprint contour,

b) specify an automobile velocity,

c) match a rolling road resistance coefficient,

d) take into account of wind velocity component oriented along the road, etc.

The facts and regularities ascertained by modelling and described above may be helpful in designing of WIM systems. In particular, it may be used as a starting material for formulation of the target inverse problem namely estimation of loads on individual axles of a motor vehicle passed over the WIM sensor.

\section{Response of Seismic Sensor Signal Modelled by the $2^{\text {nd }}$ Order Electrical Circuit}

From the theory of deformable wheel bearings [12-16] that the wheel side of the road is normal and tangential distributed reaction. Complete description of the dynamics of the mass of the vehicle forms a unified system of differential equations that take into account the interaction of two masses - rotating and moving steadily. The total number of second order differential equations is six, two for each spatial coordinate. Into account for the problem of determining the mass car all times, the drag coefficient of the road, rolling friction, etc. unreal.

Simplifying the equations of motion of a deformable wheel go to the single-mass oscillation system that takes into account only the motion of the unsprung masses in the vertical plane. Assuming that the motion is on the way to the harmonic micro profile, we obtain [14]:

$\frac{d^{2} z}{d t^{2}}+2 \alpha \frac{d z}{d t}+\omega_{n}^{2} \cdot z=\omega_{n}^{2} \cdot \varepsilon_{\text {tire }} \cdot q_{0} \cdot \cos v t$ 
Here $z$ is the coordinate of vertical movement of the masses, $\alpha=\frac{K_{a}}{2 m_{2}}$ is coefficient of damping of the unsprung masses, $m_{2}$ is unsprung weight of the vehicle, $\omega_{n}=\sqrt{\left(C_{b r}+C_{t i r e}\right) / m_{2}}$ is natural frequency of the unsprung masses, $C_{b r}$ is suspension's stiffness, and $C_{\text {tire }}$ is stiffness of the tire, $\varepsilon_{\text {tire }}=C_{\text {tire }} /\left(C_{\text {tire }}+C_{b r}\right)$ is smoothing factor of the tires $\left(\varepsilon_{\text {tire }} \approx 0,6\right), q_{0}$ is maximum height of bumps in the road, and finally, $v$ is the frequency of forced harmonic tremors from the road surface.

Try to solve the problem of simulation of this equation in the form of a circuit with an unknown input to characterize the effect of mass, friction, stiffness of tires and suspension, etc. The first attempt to determine the mass of the vehicle and the frictional forces acting on the wheel is a WIM-signal approximation in the basis of certain mathematical functions. Signal approximation by polynomials of various degrees and identify their coefficients depending on the weight and friction gives not a positive result. In the harmonic approximation basis also found no linear relationship between the weight of the car and the parameters of the harmonic series, however, possible to determine the "own" the frequency of the car on the WIM-signal, which eventually led to the idea to try to solve the problem directly in the basis of differential equations.

In the first phase was implemented simulation WIM-signal with RLC-circuit of the second order (Fig. 9) with external action in the form of a trapeze with controlled quantities of the "front", "fall", the flat top and amplitude.

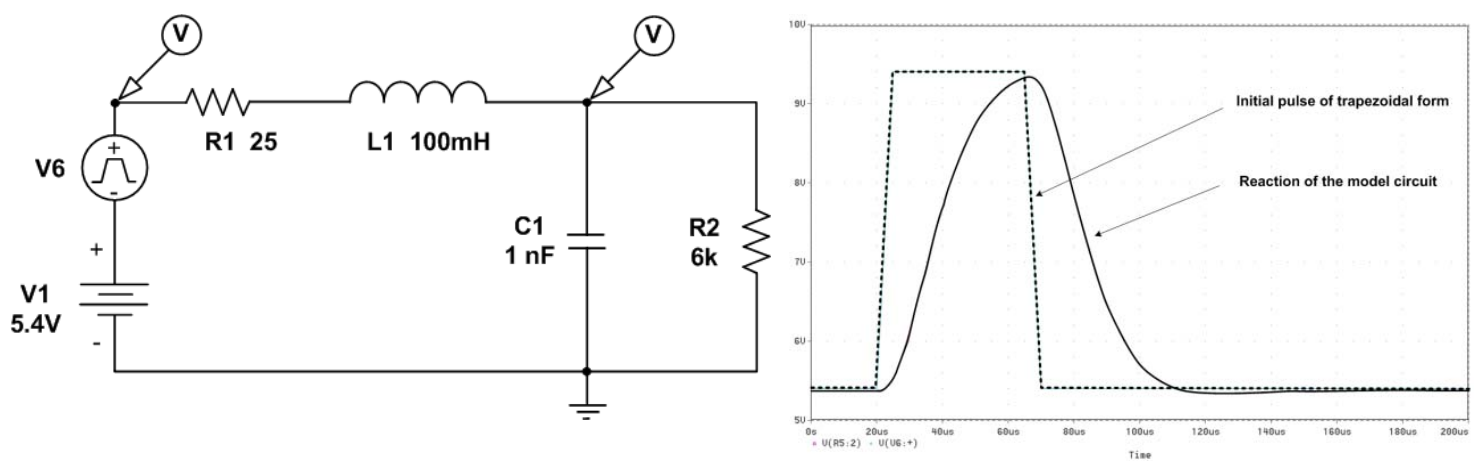

Figure 9. Simulation of WIM-signal as output reaction of the second order circuit

The figure shows part of the output response and the external action in the form of trapezoidal (Fig. 9). Since the output response is like to WIM-signal, we can try to find a relationship between the "mechanical" parameters of differential equations of the theory of deformable rolling wheels and its "electric" counterparts, including the weight of the car with the numerical values of the circuit elements.

However, there is much more productive idea to direct solution of linear (and perhaps non-linear) differential equations with forcing action in trapezoidal (or other shape). If the output variable of the equation to approximate the WIM-signal, optimising the parameters of the differential equation and find the relationship between the mass of the vehicle and the characteristics of the driving influence, the problem will be solved.

Among MATLAB program was written for solving differential equations with forcing input action in the form of a trapezoid. The objective function with a weighted least square value and the maximum deviation between the WIM-signal and the output response of the differential equation was formed. A program of "global" to minimize the objective function was designed, since the attempts to use existing local methods in MATLAB not give the desired results. This approach was applied to the real signals obtained from measurements carried out by the project "Fibre optic sensor applications for automatic measurement of the weight of vehicles in motion: research and development (2010-2012)".

\subsection{Data Processing Based on Optimisation of Modelling Input Pulse}

In a series of picture below (Figure 10), as an example, is shown the right track WIM-signal s4_70km_20_04_2012 and the results of optimisation for each wheel trucks with known weight for axes in the static (in coordinates: seconds, volts). The objective functions before and after optimisation $f 0$ and $f 00$, and the vector of "optimal" parameters $x 00$, including the values of breakpoints for trapezium and coefficients of the differential equation of second order are printed too. 

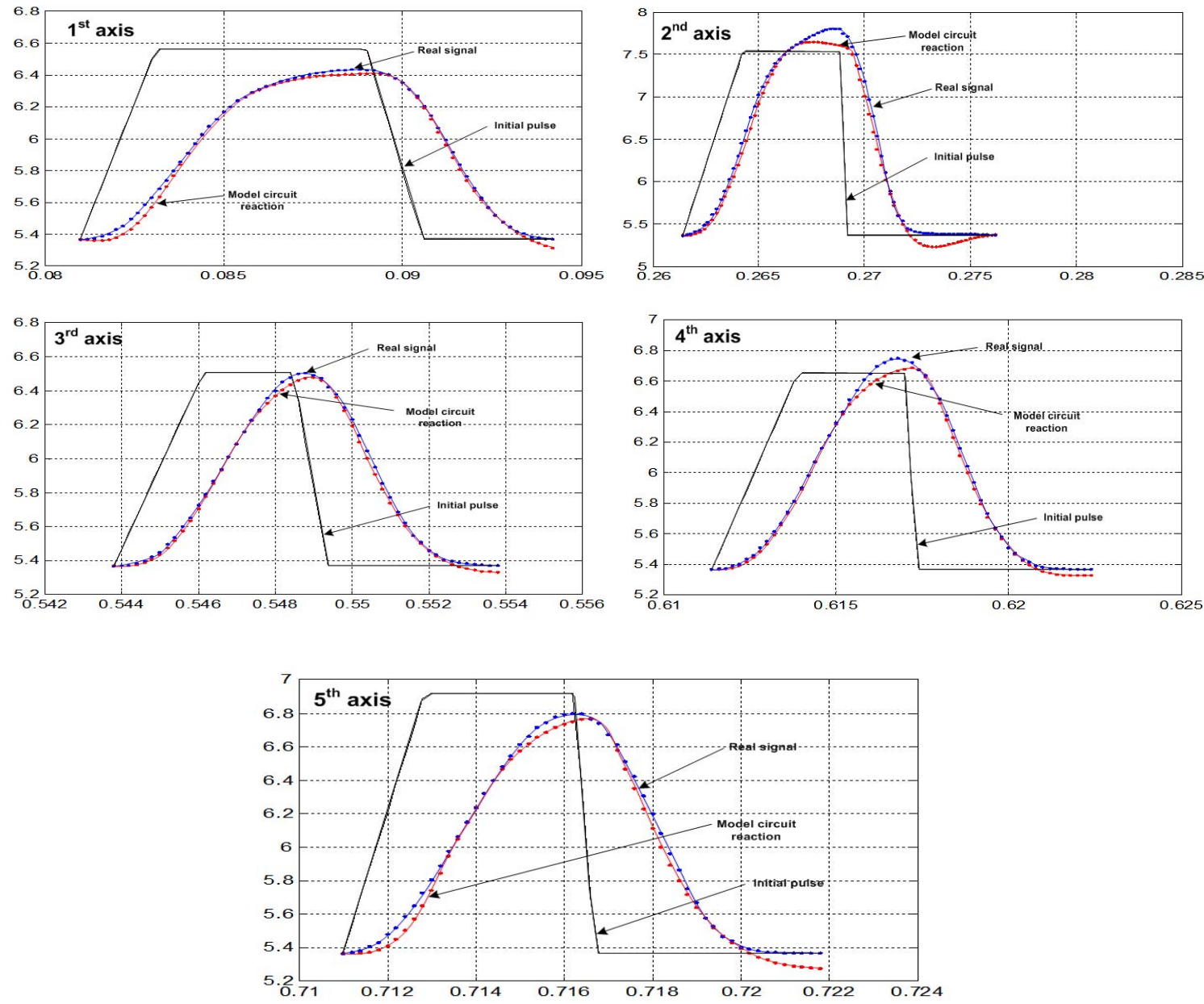

Figure 10. WIM-signal (right wheel), "optimal” output reaction and trapezoid input actions

The results of calculations based on global optimisation algorithm are as follows:

$1^{\text {st }}$ axis. $f 0=1.9415 e+001, \quad f 00=3.8514 e-001, \quad x 00=2.1339 e+000$,

$2^{\text {st }}$ axis. $f 0=5.3321 e+001, \quad f 00=1.2289 e+000, \quad x 00=2.8267 e+000$,

$3^{\text {st }}$ axis. $f 0=1.6977 e+001, \quad f 00=3.2862 e-001, \quad x 00=2.3577 e+000$,

$4^{\text {st }}$ axis. $f 0=2.5870 e+001, \quad f 00=4.3931 e-001, \quad x 00=2.5150 e+000$,

$5^{\text {st }}$ axis $f 0=1.7233 e+001, \quad f 00=6.9491 e-001, \quad x 00=1.9662 e+000$.

Initial approximation, the vector $\boldsymbol{x} \boldsymbol{0}=\{\boldsymbol{t} \mathbf{1}, \boldsymbol{t} \mathbf{2}, \boldsymbol{t} \mathbf{3}, \boldsymbol{u} \mathbf{1}, \boldsymbol{d} \mathbf{1}, \boldsymbol{d} \mathbf{2}\}$ with the values of the front, flat top and fall trapeze $\boldsymbol{t} \boldsymbol{1}=\mathbf{0 . 5}, \boldsymbol{t} \boldsymbol{2}=3, \boldsymbol{t} \mathbf{3}=\mathbf{1}$, representing the height of the trapezoid $\boldsymbol{u}_{\boldsymbol{1}}=\boldsymbol{U}_{\max }$ and the coefficients of the differential equation $d 1=1, d 2=2$, (in (9) $2 \alpha=d 1+d 2, \omega_{n}^{2}=d 1 * d 2$ )), is the same for all axes. Note that the actual parameter $d 1=0.01 * d 1$.

\subsection{Calculation of the Axle Mass Based on Results of Optimisation}

The next task is to process the data using different speeds of vehicles with known mass and finds the correspondence between the parameters of trapezoidal signal, the coefficients of the differential equation and the mechanical parameters - mass, friction force, stiffness of tires and bracket, etc. Known mass to the right track of the axes in the static are $\boldsymbol{m}=\{3680,6380,2980,2830,2955\}(\mathrm{kg})$.

We use the known mass of the $1^{\text {st }}$ axis to determine the unknown values in (9): 
$K a=(d 1+d 2) \cdot m$,

$\omega_{n}^{2}=d 1 \cdot d 2=\left(C_{b r}+C_{\text {tire }}\right) / m$.

From $(d 1=1.2039 / 100 ; d 2=1.9248 ; m=3680 \mathrm{~kg})$ we obtain $\boldsymbol{K a}=(\boldsymbol{d} 1+\boldsymbol{d} 2) * \boldsymbol{m}=\mathbf{7 1 2 3 . 9 k g}$, and $\boldsymbol{C}_{b \boldsymbol{t}}=\boldsymbol{C}_{\boldsymbol{b r}}+\boldsymbol{C}_{\text {tire }}=\boldsymbol{m} \cdot(\boldsymbol{1}+\boldsymbol{d} \boldsymbol{1} \cdot \boldsymbol{d} 2)=3765.2 \mathrm{~kg}$. We apply these factors (such as calibration) to determine the mass of 1 -axis for the signal $s 2 \_50 \mathrm{~km} \_20 \_04 \_2012(d 1=0.025769 ; d 2=1.3015)$. Now we obtain

$m 1=K a /(d 1+d 2), m 1=5.3673 e+003$.

$m 2=C b t /(1+d 1 * d 2), m 2=3.6431 e+003 .(S 2-m=3682 \mathrm{~kg})$.

The coefficients $\boldsymbol{K}_{\boldsymbol{a}}$ and $\boldsymbol{C}_{\boldsymbol{b t}}$ for $5^{\text {th }}$ axis $(d 1=0.0032466$; $d 2=1.5831 ; \mathrm{m}=2830 \mathrm{~kg}) \mathrm{cfn}$ be calculated as:

$K_{a}=(d 1+d 2) * m=4.4894 e+003, \quad C_{b t}=(1+d 1 * d 2) * m=2.8445 e+003$.

We apply these factors to determine the mass of the $5^{\text {th }}$ axis signals:

$s 2 \_50 \mathrm{~km} 20 \_04 \_2012(d 1=0.0083565 ; d 2=1.8494) m 1=2.4165 e+003, m 2=2.8013 e+003$.

$(\mathrm{S} \overline{2}-\mathrm{m}=2 \overline{8} 80 \mathrm{~kg})$

$s 1$ 70km_20_04_2012 $(d 1=0.000120 ; d 2=1.5898) m 1=2.8237 e+003, \quad m 2=2.8440 e+003$.

$(\mathrm{S} \overline{1}-m \overline{-}=2 \overline{8} 80 \overline{\mathrm{kg}})$

s3_90km_20_04_2012 $(d 1=0.039366 ; d 2=1.1662) m 1=3.7239 e+003, \quad m 2=2.8196 e+003$.

$(\mathrm{S} \overline{3}-\mathrm{m}=29 \overline{6} 5 \mathrm{~kg})$

It is seen that the obtained value is closer to the mass $\mathrm{m} 2$ and is located to an accuracy of less than $5 \%$. Let's verify these findings for the third axis, taking the "calibration" signal: s4_70km_20_04_2012 $(d 1=4.5769 e-004 ; d 2=1.4913 e+000 ; m=2980 \mathrm{~kg})$.

Then $K_{a}=(d 1+d 2) * m=4445.4 \mathrm{~kg} ; C_{b t}=(1+d 1 * d 2) * m=2982 \mathrm{~kg}$ and the mass of the third axis for the various signals is
1. s2 50km_20_04_2012,
$m \overline{1}=2.4672 e+003$,
$(d 1=4.0361 e-003$
$d 2=1.7978)$
$m 2=2.9605 e+003 ; \quad(m=3045 \mathrm{~kg})$
2. s3_50km_20_04_2012,
$m \overline{1}=1 . \overline{62} 3 \overline{7 e}+\overline{003}$,
$(d 1=1.8952 e-003$
$d 2=2.7360)$
$m 2=2.9666 e+003$;
$(m=2900 \mathrm{~kg})$
relative error $=-2.7750 \mathrm{e}-002$,
s3 90km_20_04_2012,
$m \overline{1}=1 . \overline{1} 16 \overline{1 e}+\overline{003}$,
$(d 1=1.1673 e-002$;
$m 2=2.8949 e+003 ;$
$d 2=2.5788)$
relative error $=-2.2966 \mathrm{e}-002$,
4. s1_70km_20_04_2012,
$m \overline{1}=2 . \overline{6475 \bar{e}}+\overline{003}$,
$(d 1=7.9706 e-003$
$(m=2900 \mathrm{~kg}), \quad$ relative error $=-1.7586 \mathrm{e}-002$,
$m 2=2.9428 e+003$
$d 2=1.6711)$
5. s5 $70 \mathrm{~km} 20 \quad 04 \quad 2012$,
$m \overline{1}=2 . \overline{29} 1 \overline{0 e}+\overline{003}$,
$(d 1=7.1044 e-003$
$(m=2945 \mathrm{~kg}), \quad$ relative error $=-7.4703 \mathrm{e}-004$,
$m 2=2.9416 e+003$
$d 2=1.9333$
$(m=2967,5 \mathrm{~kg}), \quad$ relative error $=-1.1545 \mathrm{e}-003$.

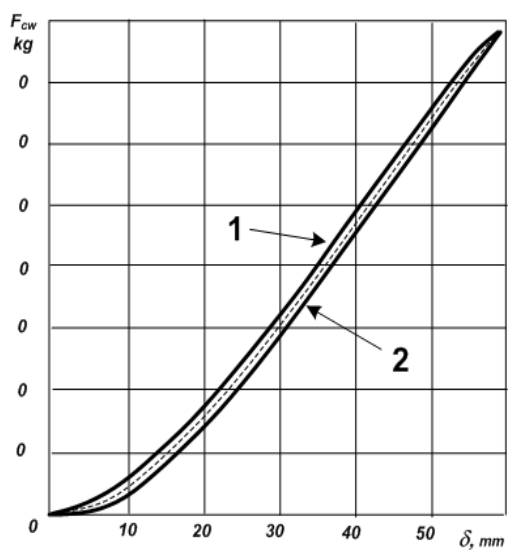

Figure 11. Elastic characteristics of the tire air pressure is $3 \mathrm{~kg} / \mathrm{cm}^{2}$ (here $\boldsymbol{F}_{\boldsymbol{c} w}$ is an elastic force in tires), and 1 - loading, 2 - unloading curves [14] 
So, as it was seen, in all of calculations, the relative error wasn't exceeding 3\% of measured weight's value but it isn't the limit. For example, it is a graph (Figure 11) for the ZIL-130 truck vehicle's tires, noting that the damping capacity of the tire due to intermolecular friction rubber and internal friction between the elements of the tire [14]. The presence of friction leads to the hysteresis loop on the elastic characteristics of the tire, which becomes narrower with increasing velocity.

The knowledge of static stiffness values for the currently operating commercial vehicles would be helpful for implementation of the results of this approach or its modified version into weight-in-motion (WIM) technologies because of high accuracy (till 3\%) of it.

\section{Problem of Decision Making Concerning the Overloaded Vehicle Selection}

\subsection{Formalization of the Decision Making Problem}

One of the problems of the dynamic weighing is the task of the selection of the overloaded vehicles in the traffic flow. In this case, the weighing task will be transformed into the decision making task - if the vehicle is overloaded or not. The decision-making problems are usually solved by comparing the current event with some the so-called reference one.

In the systems with electronic sensors, the standard of reference can be specified either in the form of the reference signal, or as a device, which carries the characteristics of the reference signal. In the dynamic weighing systems where fibre optical sensors are used, the reference signal from the sensors' output should have the characteristics that correspond to the true weight of the vehicle detected by the sensor. As there are random and regular destabilizing factors that distort the signal from the fibre optical sensors' output, the reference signal can only be formed as the result of the averaging signals from the output of the same sensor due to repeated overriding of the vehicle loaded with the reference weight. Thus, the reference signal - this is the response of the sensor in some measuring system, that corresponds to the averaged response of the sensor of the real system to the repeated overriding of the vehicle loaded with the reference weight. The corresponding conditional system will be called - the reference system.

After the reference system is created, the decision making tasks concerning the vehicles' accordance to the allowable weight for a given road can be made, for example, by the correlation analysis technique [25]. This calls to set the model of the reference system and to set its parameters in such a way, that the output signal would have characteristics that corresponds to the maximum of the allowable weight of the vehicle for the particular type of the road. Comparing the responses from the sensors' output of the real system, when the sensor detects the passing vehicle only once, with the response at the reference systems' output, using the correlation method, it is possible to determine what event rate is bigger - has the vehicle the greater weight than existed in the reference system or the smaller one.

\subsection{Reference Dynamic Weighing System and its Features}

As stated above, the decision making task concerning the vehicles' overloading can be solved by comparing the sensors' response of the real systems' output with the sensors' response of the reference system. Besides, the sensors' response of the reference system should be in accordance with the average response of the real systems' sensor to the repeated overriding of the vehicle loaded with the reference weight. The creation of the adequate reference system is a complex technical challenge that requires taking into account all the destabilizing factors that mentioned above, which affect the WIM sensors. And, as among the destabilizing factors there are vehicle specifications that lead to the errors in the sensors' readings, the design of the reference system can only be applied to the specific and pre-selected vehicle.

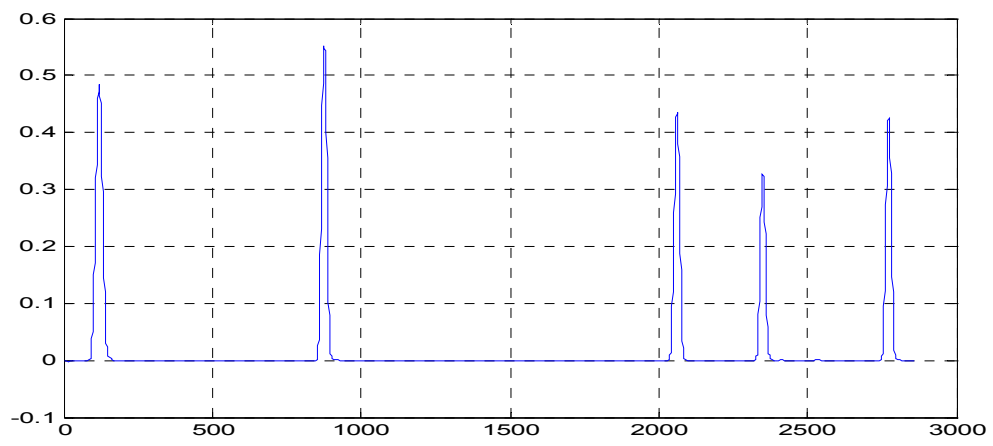

Figure 12. The response of sensors' output, when the empty truck passes it at the speed of $90 \mathrm{~km} / \mathrm{h}$ 
From the figure, showed above, it is possible to see, that when the truck passes through the sensor placed into the road, each of the five axles of the vehicle appears at the sensors' output as leap of the voltage (vertical axis). The set of five leaps of the voltage on the time axis (the horizontal axis shows the numbers of discrete signal samples in time) is the system response from the passage of the selected vehicle type.

It is also can be seen that the responses of the sensor while passing the empty vehicle and the loaded vehicle, with the reference weight, are different. However, the degree of the responses similarity or difference can be determined statistically only. The procedure of the comparison of responses is complicated by the fact that the speed of the vehicle when driving over a sensor is not known in advance and, therefore, the location of the voltage leaps on the time axis is unknown. These circumstances brings us to the need of the data pre-processing in the fibre optical sensor, for the purpose of bringing the sensors' output responses into the one and the same time frame. As well, in the same time frame should be generated the reference systems' response from the vehicle of the given type, that is loaded with the reference weight and that passes the sensor. The device that generates this reference response is the reference system.

To bring the response from the sensors' output into the same time frame, it is needed to make the following calculations:

- Calculation of the centre of mass of statically weighed vehicles of a given type.

- Calculation of the centre of mass of dynamically weighed vehicles of a given type.

- Calculation of the speed of the vehicle of a given type.

Basing on the assessment of the decentralization of the mass of the dynamically weighed vehicle with respect to the centre of mass of the statically weighed vehicle, and, knowing the speed of the latter, the peak voltage amplitude response is recalculated from the sensors' output in the new coordinate system, which corresponds to the single time frame. To reduce the errors associated with the temperature sensor instability, during the recalculation of the peak voltage amplitude response from the sensors' output, there are calculated and introduced the correction coefficients. The transformation procedure to the same time frame of the WIM sensors' response is sufficiently completely described in [26].

\subsection{Reference Signal Normalization and the Conception of the Reference System}

As the reference signal - it should be the response from the WIM sensors' output that is normalized to the common time frame, and contains the information about the vehicles' weight that passes the sensor. The reference signal is generated by the averaging the values of the voltage peaks' amplitudes response from the sensors' output resulting from the repeated calibrating overriding of the specified vehicle type with the reference load.

In this case, for the averaging procedure it is very important to know, how many sensors are installed for being passed by the vehicle, or how many times the vehicle passes the sensors, all of this will result the averaging. Thus, there is an opportunity to get two signals in one time frame. The first signal corresponds to the distribution of the voltage peaks' amplitudes of the statically weighted vehicle, and the second one - to the distribution of the voltage peaks' amplitudes in the reference signal.

The work of the WIM, in this case, can be interpreted as the work of some equivalent electronic system. At the input of this system was sent the reference signal and the response of this system is the signal that corresponds to the distribution of the voltage peaks' amplitudes of the statically weighted vehicle of the specified type and with the reference load. In what follows, this system will be called - the reference system.

Main element of the reference system is the filter with the finite impulse response, which transforms the reference signal into the corresponding distribution of the peaks' amplitudes of the statically weighted vehicle of the specified type and with the reference load. The quality (accuracy) of the transformation depends on the correctness of the definition of the filters' weight coefficients. Due to the complexity of the exact analytical description of the initial signals it was offered to receive the adequate value of the filters' weight coefficients by the method of systems' identification [25].

On Figure 13 the structure of the algorithm is shown for defining the characteristics of the reference system. Main element of the structure is the filter with variable weight coefficients (the transversal filter). On the filters' input the reference signal is sent. The task of the algorithm is following: to minimize the error of the reference signal reproduction by adjusting the parameters of the transversal filter. As the reference signal, the signal was taken, that corresponds to the distribution of the voltage peaks' amplitudes of the statically weighed vehicle of the specified type and with the reference load. 
To obtain the weight coefficients of the transversal filter were used the least squares method [25]. This adaptive algorithm was taken due to the fact that it works in real time, i.e., there is no need to accumulate errors from the sensors. The use of other adaptive algorithms, such as normalized LMS, variable step size LMS and sign algorithm LMS, do not lead to the significant improvements in the calculation of weight coefficients.

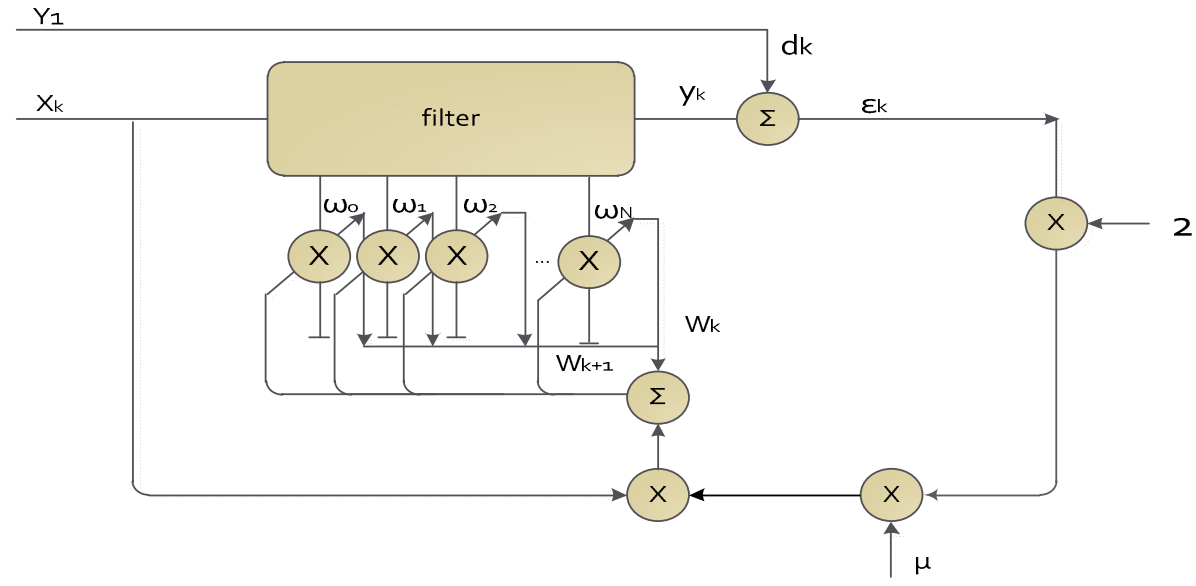

Figure 13. The structure of the adaptive algorithm for determining the weight coefficients of the transversal filter

Calculation of the coefficients is carried out according to the formula (12)

$w(k+1)=w(k)+2 \mu \cdot e(k) \cdot u(k)$,

where $\mu$ is positive coefficient, which is called the size of the step, $e(k)$ is the replication error of the reference signal, $u(k)$ is the content vector of the delay-line on the $k$-step. Later, these weight coefficients are stored in the form of the constant filters' weight coefficients of the reference system.

\subsection{Overloaded Vehicles' Identification Results}

During the test of the proposed algorithm there were formed two reference systems (for the reference signal of the vehicle that is fully loaded; and for the reference signal of the empty vehicle.

Table 1.

\begin{tabular}{||c|c|c|c|c|c||}
\hline \multirow{2}{*}{ Vehicle passing options with different weights } & \multicolumn{2}{c|}{$\begin{array}{c}\text { Discrete filter Nr.1 } \\
\text { (reference of empty vehicle) }\end{array}$} & \multicolumn{2}{c||}{$\begin{array}{c}\text { Discrete filter Nr.2 } \\
\text { (reference of loaded vehicle) }\end{array}$} \\
\cline { 2 - 6 } & & $\begin{array}{c}\text { Speed, } \\
\mathbf{7 0 k m / h}\end{array}$ & $\begin{array}{c}\text { Speed, } \\
\mathbf{9 0} \mathbf{~ k m} / \mathbf{h}\end{array}$ & $\begin{array}{c}\text { Speed, } \\
\mathbf{7 0} \mathbf{~ k m} / \mathbf{h}\end{array}$ & $\begin{array}{c}\text { Speed, } \\
\mathbf{9 0 k m} / \mathbf{h}\end{array}$ \\
\hline \multirow{3}{*}{ Number of empty vehicle passing } & 1 & 0.0983 & 0.1044 & 0.5499 & 0.4444 \\
\cline { 2 - 6 } & 2 & 0.1273 & 0.0740 & 0.5923 & 0.4093 \\
\cline { 2 - 6 } & 3 & 0.1051 & 0.0725 & 0.5605 & 0.4084 \\
\cline { 2 - 6 } & 4 & 0.0602 & 0.0719 & 0.5023 & 0.4081 \\
\hline \multirow{3}{*}{ Number of loaded vehicle passing } & 1 & 0.4294 & 0.2410 & 0.2018 & 0.2186 \\
\cline { 2 - 6 } & 2 & 0.3864 & 0.3436 & 0.1483 & 0.0568 \\
\cline { 2 - 6 } & 3 & 0.3250 & 0.3263 & 0.2204 & 0.0878 \\
\cline { 2 - 6 } & 4 & 0.5113 & 0.3436 & 0.1167 & 0.0568 \\
\hline
\end{tabular}


The converted signal from the fibre optical sensors' output was sent to the discrete filters of the corresponding reference systems, and there were estimated the relative standard mean square errors on the filters' outputs. In the Table 1, there are shown the following results that were received during the working process of the algorithm of the systems identification.

As one can see, not all the results are well-defined. This could happen due to the fact that the fibre optical sensor was not tested for the spatial homogeneity of the sensor response over the period of the working zone. In the data used, the possible errors can occur. This is associated with the fact that the signals were not recorded in one day, but for a certain period of time, when the connection and the disconnection of the equipment from the sensors were placed. According to the results, it is possible to conclude that the designed system copes with the task of the vehicles' identification by the excess of the permitted maximum load on the road.

\section{Weighting-In-Motion of the Axles of Vehicle by the Area Method}

\subsection{WIM Basic Characteristics}

The fibre optic force sensor is a cable consisting of a photoconductive polymer fibres coated with a thin light-reflective layer (Fig. 14). A light conductor is created in this way, from which the light cannot escape. If you direct a beam of light to one end of the cable, it will come out from the other end, and in this case the cable can be twisted in any manner. In order to measure the force acting on the cable, the amplitude technology is more appropriated for measurement, when measures the optical path intensity, which changes while pushing on the light conductor along its points.

At these points the deflection of a light conductor and reflective coating occurs, that is why the conditions of light reflection inside are changed, and some of it escapes. The greater the load - the less light comes from the second end of the light conductor. Therefore the sensor has the unusual characteristic for personnel, familiar with strain gauges: the greater the load - the lower the output is. Apart from the fact that it is reversed and in addition to this it is non-linear.

Let to avoid the inaccuracy of zero load level we need to exclude the high frequency components from the voltage signal at the output of the sensor's transducer by filtering as well as to recalculate the voltage signal $\boldsymbol{U}(\boldsymbol{t})$ (Fig. 14(a)) into the relative visibility losses signal $\boldsymbol{V}(\boldsymbol{t})$ (Fig. 14(b)), directly related to the weight pressure on the FOS surface, by the transformation (13):

$\boldsymbol{V}(\boldsymbol{t})=\frac{\boldsymbol{U}_{0}-\boldsymbol{U}(\boldsymbol{t})}{\boldsymbol{U}_{0}}$,

where $\boldsymbol{U}_{\boldsymbol{0}}$ is the voltage of sensor output with zero load. The signal transformation to the relative visibility losses signal $\boldsymbol{V}(\boldsymbol{t})$ gives the possibility to compare signals for different measurements in different conditions.

Fibre optic load-measuring cables are placed in gap across the road and are filled with resilient rubber (Fig. 14). The gap width is $30 \mathrm{~mm}$. Since the sensor width is smaller than the tyre footprint on the surface, the sensor takes only part of the weight axis. Two methods are used in the existing systems to calculate the total weight of the axle [17, 27]: the Basic Method and the Area Method. The following formula is used to calculate the total weight of the axis using the Basic Method:

$$
W_{h a}=A_{t} \cdot P_{t},
$$

where $\boldsymbol{W}_{\boldsymbol{h} \boldsymbol{a}}$ is the weight on half-axle, $\boldsymbol{A}_{\boldsymbol{t}}$ is the area of the tyre footprint, and $\boldsymbol{P}_{\boldsymbol{t}} \sim \boldsymbol{V}(\boldsymbol{t})$ is the air pressure inside the tyre and, according to Newton's $3^{\text {rd }}$ law, it is proportional to the axle weight.

As we can see the exact values of the formula factors are unknown. The area of the tyre footprint is calculated roughly by the length of the output voltage impulse, which, in its turn, depends on the vehicle speed. The Area method uses the assumption that the area under the recorded impulse curve line, in other words - the integral, characterizes the load on the axle. To calculate the integral, the curve line is approximated by the trapezoid. In this case the smaller the integral - the greater the load. This method does not require knowing the tyre pressure, but it requires the time-consuming on-site calibration. Also, it has to be kept in mind that the time of the tyre passing on the sensor is too small to get an electrical signal of high quality for its further mathematical processing. We use the Area method for footprint area definition in (14), but the pressure is measured from the signal amplitude. 

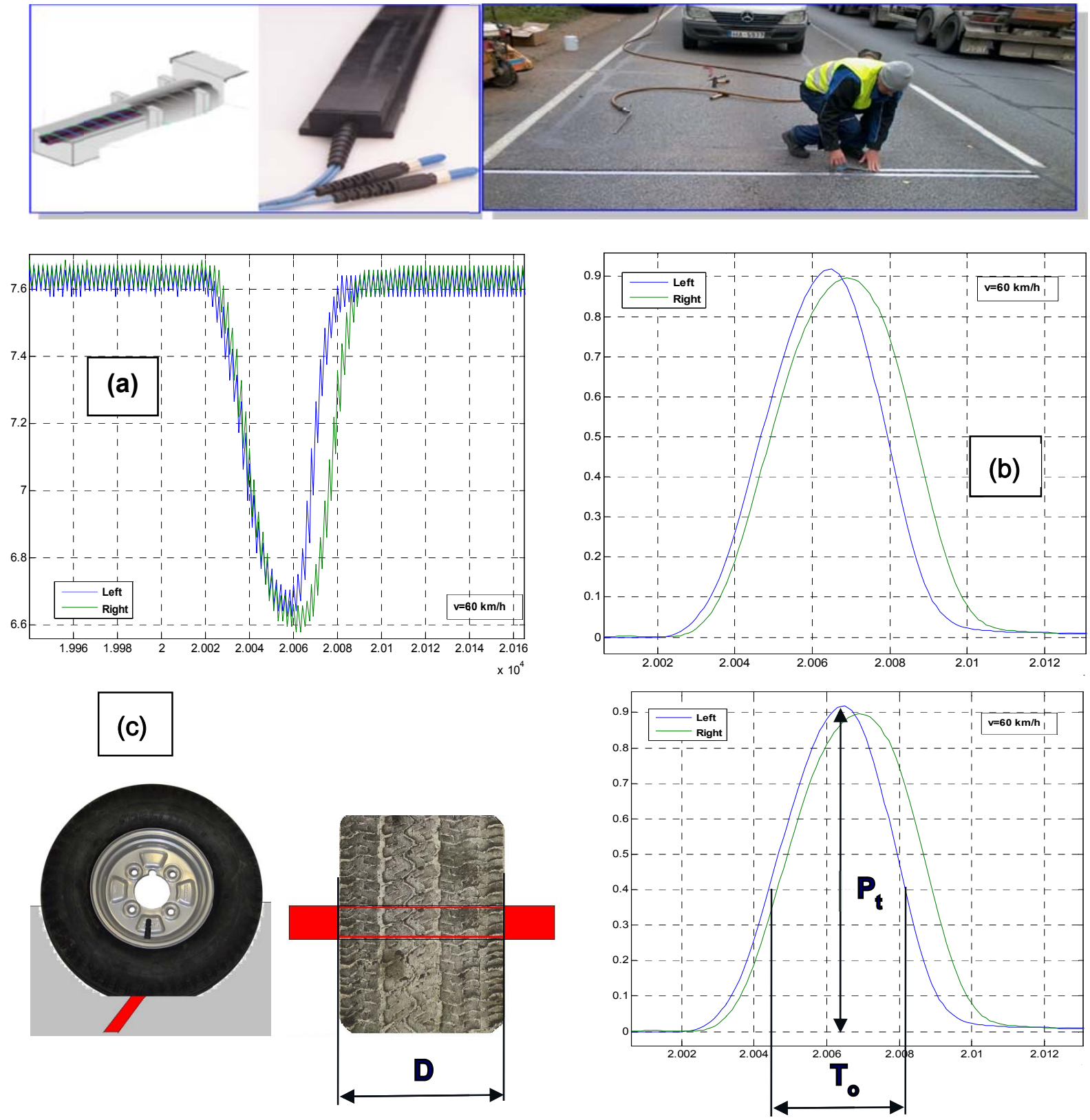

Figure 14. Location and waveform of the SENSORLINE PUR fibre optic sensor [28], (a) Voltage, (b) Visibility losses, (c) sensor's position against the wheel and wheel's footprint, (d) measured parameters for weight calculation

\subsection{Field and Laboratory Experiments with FOS and Weighting-In-Motion Results}

There was the set of measurement experiments with the roadside FOS sensors on April, 2012. Loaded truck (Fig. 15) was preliminary weighed on the weighbridge with the accuracy $<1 \%$.

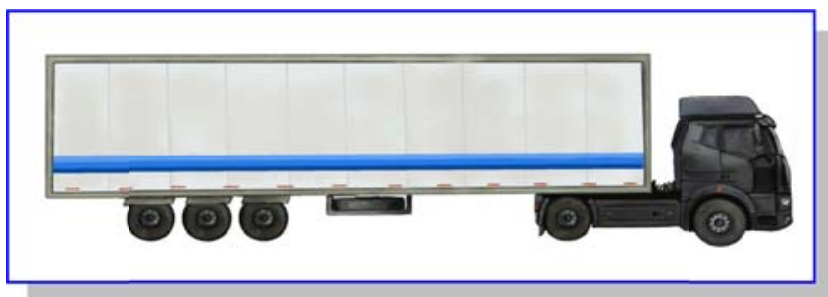

Figure 15. Experimental truck "Volvo FH12" with full load $36900 \mathrm{~kg}$ 
Table 2.

\begin{tabular}{|c|c|c|c|c|c|}
\hline \multicolumn{6}{|c|}{ Date: 20.04 .2012 (Air Temperature $+12^{\circ} \mathrm{C}$ ) } \\
\hline $\begin{array}{c}\text { Etalon axle's weight } \\
\text { (tons): }\end{array}$ & 7.296 & 12.619 & 5.509 & 5.641 & 5.844 \\
\hline
\end{tabular}

Etalon weights of separate axles are given in the Table 2. The signals from output of FOS sensors for truck speed $70 \mathrm{~km} / \mathrm{h}$ and $90 \mathrm{~km} / \mathrm{h}$ are demonstrated on Figure 15 . It is evident that the signals for different speed are strongly changed by amplitude and the proportion of amplitudes does not fit the axle's weights (Fig. 16). The reason of this behaviour may be concluded in the FOS properties such as weight (pressure) distribution along the sensor's length as well as sensor's non-linearity and temperature dependence.
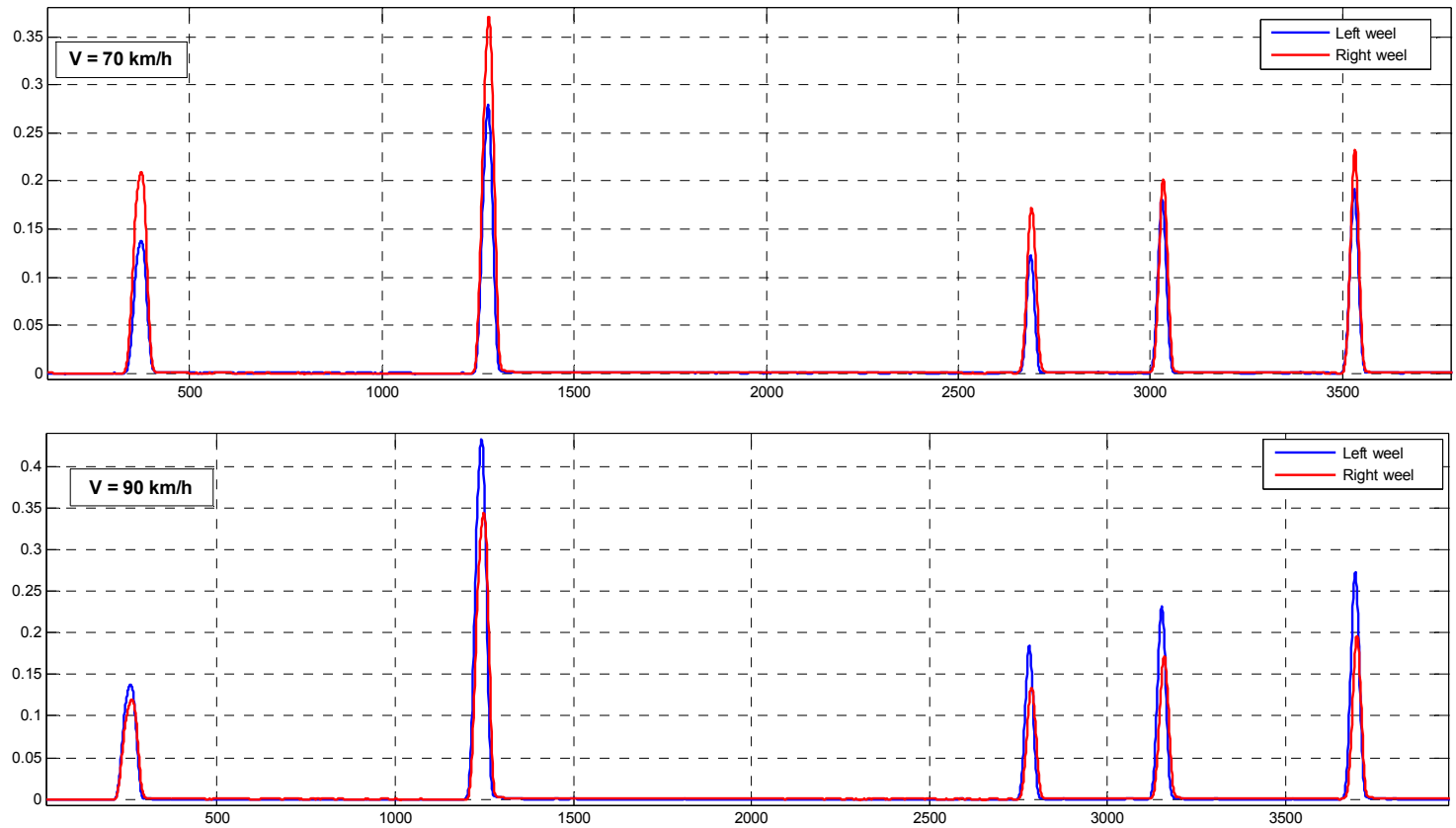

Figure 16. Examples of FOS signals of experimental truck for vehicle's speed $70 \mathrm{~km} / \mathrm{h}$ and $90 \mathrm{~km} / \mathrm{h}$ respectively

Load characteristic was measured from the SENSORLINE PUR fibre optic sensor by means of a SL Transducer (optical interface) optical signal analyser has been developed by SensorLine GmbH [28].

For obtaining the static load characteristics of the sensor the MTS compression machine of the Latvian University Institute of Polymer Mechanics was used. Effort in the range 0-2000 kg was transmitted to the sensor through a steel plate (see Figure 17 (a)) the size of $200 \mathrm{~mm}$ by $200 \mathrm{~mm}$ at the temperature conditions of $+18^{\circ} \mathrm{C}$. The area of application of the force to the sensor was $30 \mathrm{~mm}$ to $200 \mathrm{~mm}$. Load curves (gradual increase of the force) and unloading (gradual decrease) of the sensor are shown on Figure 17 (b).

The curves clearly demonstrate the presence of nonlinear behaviour - the so-called "hysteresis loop", or the difference between the curves "load-on" and "load-off". The reason for this is the residual deformation of rubber protective sensor housing. These curves are conveniently approximated by hyperbolic tangent function:

$V(p)=a_{0}+a_{1} \cdot \tanh (\omega \cdot p+\varphi)$,

where $\boldsymbol{p}$ is the pressure on the sensor's surface but $\boldsymbol{a}_{0}, \boldsymbol{a}_{1}, \boldsymbol{\omega}$ and $\boldsymbol{\varphi}$ - are the parameters of approximation. For example, "load-on" curve from the Figure 17(a) conditionally describes by the next approximation coefficients: $\boldsymbol{a}_{0}=\mathbf{0 . 4 9 9 4}, \boldsymbol{a}_{1}=\mathbf{0 . 5 0 0 6}, \boldsymbol{\omega}=\mathbf{0 . 1 4 8 0}, \varphi=\mathbf{- 3 . 3 7 0 2}$, calculated by the method of least square criteria optimisation. For the local approximation is quite suitable to use cubic or quadratic approximation polynomials, defined by the criterion of least squares. 
For example, "load-on" curve from the Figure 17(a) conditionally describes by the next approximation coefficients: $\boldsymbol{a}_{\boldsymbol{0}}=\mathbf{0 . 4 9 9 4}, \boldsymbol{a}_{1}=\mathbf{0 . 5 0 0 6}, \boldsymbol{\omega}=\mathbf{0 . 1 4 8 0}, \boldsymbol{\varphi}=\mathbf{- 3 . 3 7 0 2}$, calculated by the method of least square criteria optimisation. For the local approximation is quite suitable to use cubic or quadratic approximation polynomials, defined by the criterion of least squares.
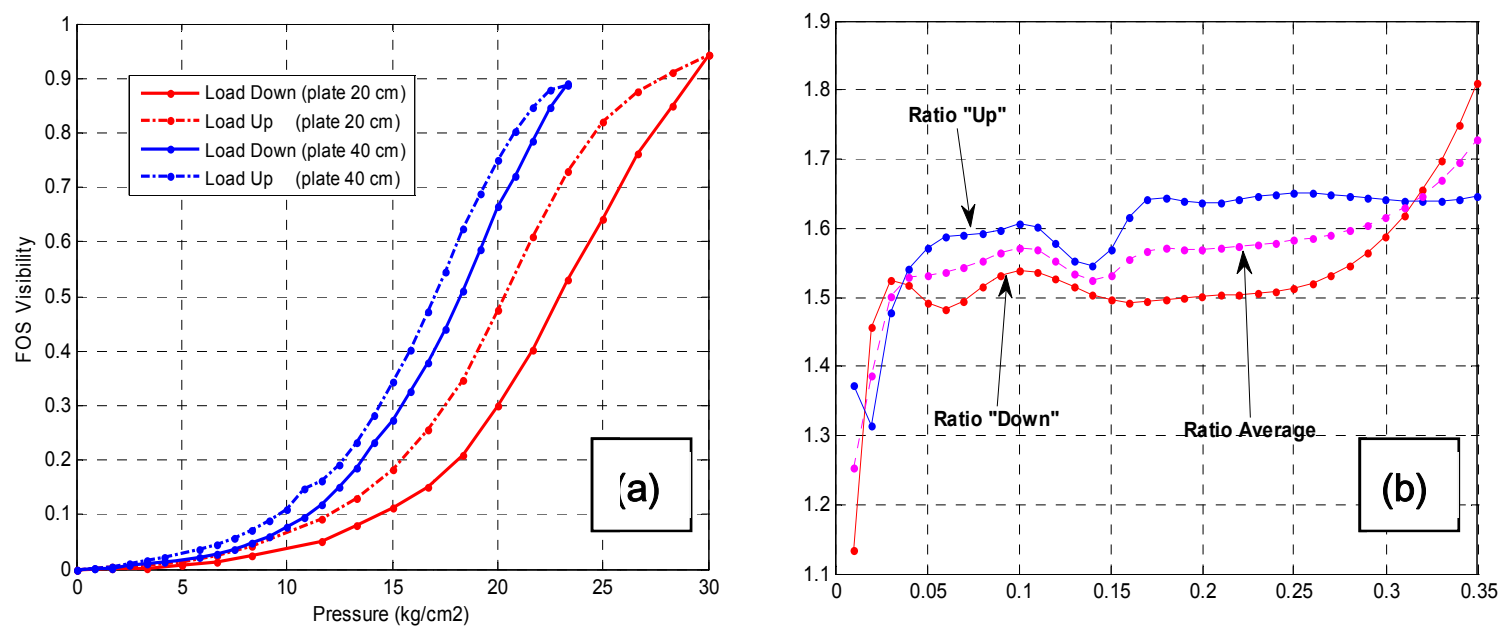

Figure 17. (a) Experimental static load characteristic of FOS with doubling of active area and (b) Ratio between two curves by the length of 200 and $400 \mathrm{~mm}$ respectively

Another experiment was associated with the influence of sensor data, produced by the dual wheels. Comparison of load curves at the impact area through the steel plate with the size of $200 \mathrm{~mm}$ by $200 \mathrm{~mm}$ and $400 \mathrm{~mm}$ by $400 \mathrm{~mm}$ is shown on Figure 17(a). The impact area on the sensor is increased by 2 times, and the losses of transparency of the FOS, as can be calculated from the relative position of the curves (Figure 17(b)), decreases approximately by $\pi / 2$ times, due to the radial distribution of load along the contact surface of the steel plate and the sensor.
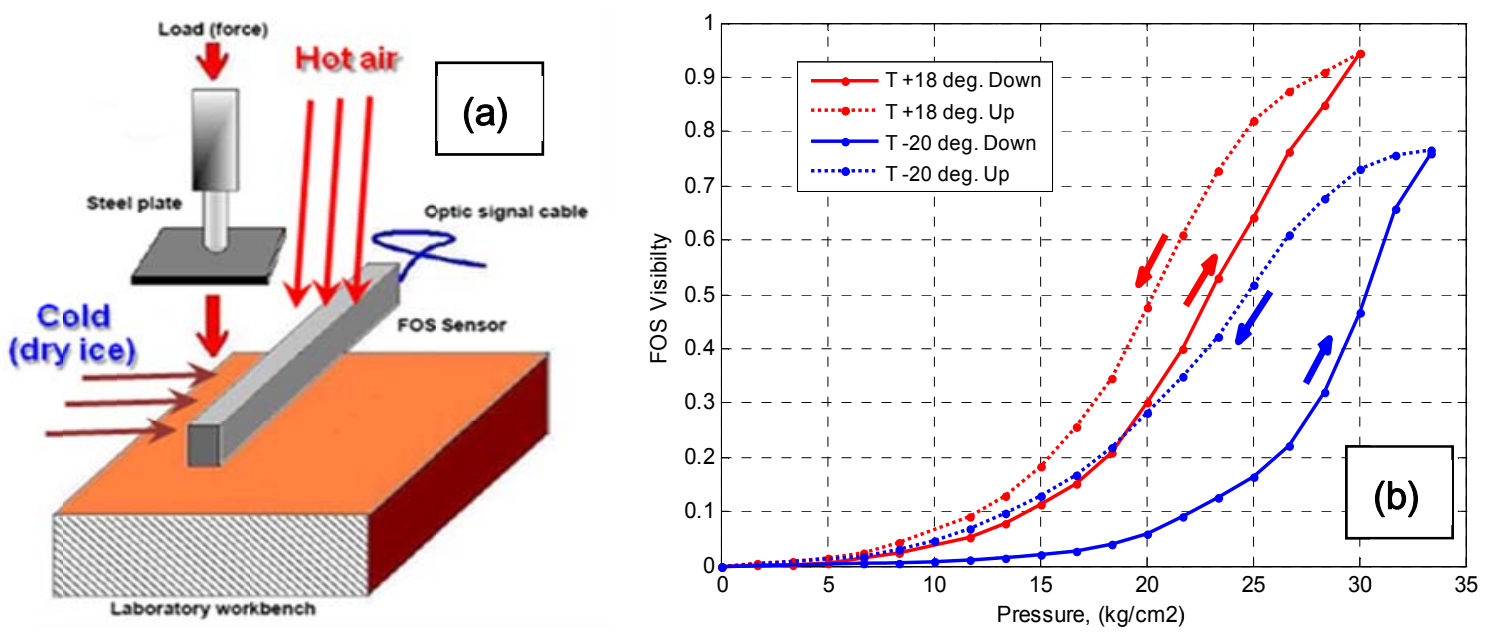

Figure 18. (a) Scheme of temperature factor measurement and (b) Experimental static load characteristic of FOS with doubling of active area of wheel's footprint

In actual conditions of use the fibre optic sensor (FOS) with the protective rubber housing is built into the road surface and, therefore, affected by temperature, which changes the stiffness of rubber. An experiment determining the effect of temperature's factor is to obtain load characteristics and comparison with FOS at $18^{\circ} \mathrm{C}$ and after cooling in the freezer camera down to $-20^{\circ} \mathrm{C}$. The results of these measurements are shown on Figure 18(b). 
Due to changes in the cover housing rubber stiffness, the measurements of pressure changes up to approximately $50 \%$ and increased residual effects (hysteresis). Consequently, it is necessary to use the temperature correction in the measuring scheme.

Another source of measurement errors can be dynamic oscillation of the load of each wheel due to movement. The transversal dynamic oscillations leads to different loads on each wheel in axle but not add the error to the common axle's weight measurement results. Most sufficient seems the longitudinal oscillation because it leads to additional vertical force (or decreasing of it) to both wheels in axle simultaneously.

For correction of these errors we assume that relative value of friction force, calculated by decomposition of the signal on even and odd parts [30], is dependent on footprint length and, respectively, it changes due to dynamic oscillations. From other hand, without oscillations it is assumed to be in proportion with the amplitude of relative derivative of the signal (rate of grow).
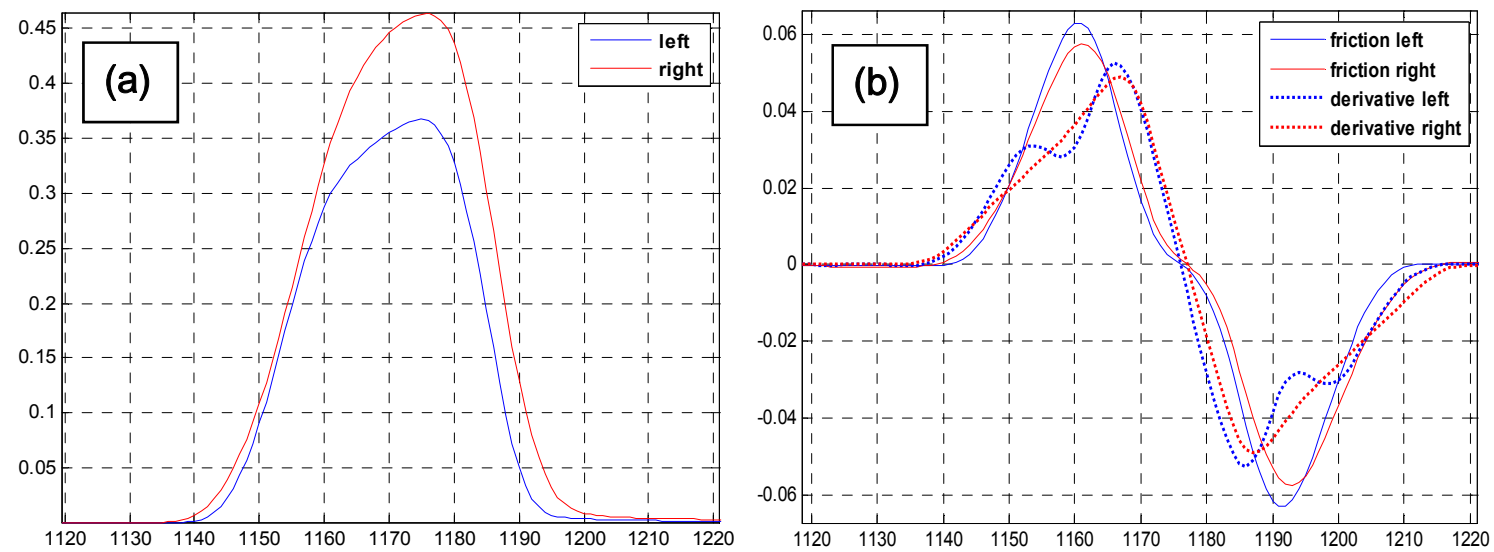

Figure 19. (a) FOS signals from one axle, and (b) relative friction and derivative components for the same signals

An example of these components of the signals is presented on Figure 19 for both left and right wheels. As it is seen from the Figure 19, the friction and derivative of weight component slightly differs to each other by the amplitude. If the $\boldsymbol{P}_{\max }$ is the amplitude of weight component's signal $\boldsymbol{P}(\boldsymbol{t})$ and $\boldsymbol{D} \boldsymbol{P}_{\max }$ is the amplitude of weight component's derivative $\boldsymbol{d P}(\boldsymbol{t}) / \boldsymbol{d t}$, then we can name the $\boldsymbol{D} \boldsymbol{P}_{\boldsymbol{N}}$ value as normalized derivatives amplitude:

$$
D P_{N}=\frac{D P_{\max }}{P_{\max }}, \quad D F_{N}=\frac{F_{\text {max }}}{S F_{\max }},
$$

$S F_{\max }=\max (S F(t))-\min (S F(t))$,

where $\boldsymbol{F}_{\boldsymbol{m a x}}$ is the maximum of friction component $\boldsymbol{F}(\boldsymbol{t})-$ see Figure 19(b), and

$S F(t)=\int_{0}^{t} F(\tau) d \tau \quad$ is the integrated friction component's function.

The idea of errors correction is in the equalization of both components for each wheel in axle, when scaled friction component again add to weight component to obtain modified signal $\boldsymbol{S}_{\text {mod }}(\boldsymbol{t})$ without influence of dynamic oscillations:

$$
\boldsymbol{S}_{\text {mod }}(\boldsymbol{t})=\boldsymbol{P}(\boldsymbol{t})+\frac{\boldsymbol{D P _ { N }}}{D \boldsymbol{F}_{N}} \cdot \boldsymbol{F}(\boldsymbol{t})
$$

Then modified signal (18) again must be under decomposition on weight (even) and friction (odd) components for weight calculation according to expression (14). The results of application of proposed approach are reflected in Table 3. 
The accuracy of weighing the vehicle in motion depends on many factors, but the main errors in the existing systems consist of the following: non-linearity and inertia of the sensor, thermal effects, and inertial force of an oscillating vehicle.

Applying the algorithm of FOS signal processing with the approximation of nonlinear characteristics of the sensor (15) for a suitable range of temperatures, it is possible to calculate the following weights of axes (Table 3):

Table 3.

\begin{tabular}{|c|c|c|c|c|c|c|}
\hline \multicolumn{7}{|c|}{ Date: 20.04.2012 (Air Temperature $+12^{\circ} \mathrm{C}$ ) } \\
\hline & alon axle's weight (tons): & 7.296 & 12.619 & 5.509 & 5.641 & 5.844 \\
\hline \multicolumn{7}{|c|}{ Speed: $10 \mathrm{~km} / \mathrm{h}$} \\
\hline No & Parameter: & $1^{\text {st }}$ axle & $2^{\text {nd }}$ axle & $3^{\text {rd }}$ axle & $4^{\text {th }}$ axle & $5^{\text {th }}$ axle \\
\hline \multirow{2}{*}{1} & Axle's weight (tons) & 8.1876 & 13.3466 & 5.0245 & 5.5659 & 5.4438 \\
\hline & Error (\%) & $12.21 \%$ & $5.77 \%$ & $-8.79 \%$ & $-1.33 \%$ & $-6.84 \%$ \\
\hline \multirow{2}{*}{2} & Axle's weight (tons) & 7.8218 & 13.5065 & 5.1057 & 5.7214 & 6.6604 \\
\hline & Error (\%) & $7.20 \%$ & $7.03 \%$ & $-7.32 \%$ & $1.42 \%$ & $13.97 \%$ \\
\hline
\end{tabular}

Speed: $20 \mathrm{~km} / \mathrm{h}$

\begin{tabular}{|c|l|c|c|c|c|c|}
\hline \multirow{2}{*}{1} & Axle's weight (tons) & $\mathbf{8 . 1 4 3 1}$ & 13.2625 & 4.9336 & $\mathbf{5 . 6 8 3 0}$ & $\mathbf{5 . 6 1 1 0}$ \\
\cline { 2 - 6 } & Error (\%) & $11.61 \%$ & $5.10 \%$ & $-10.44 \%$ & $\mathbf{0 . 7 4 \%}$ & $\mathbf{3 . 9 8 \%}$ \\
\hline \multirow{2}{*}{2} & Axle's weight (tons) & 8.3815 & 13.9084 & 4.9293 & 5.3014 & 5.2716 \\
\cline { 2 - 6 } & Error (\%) & $14.87 \%$ & $10.22 \%$ & $-10.52 \%$ & $-\mathbf{6 . 0 2} \%$ & $-9.79 \%$ \\
\hline
\end{tabular}

Speed: $50 \mathrm{~km} / \mathrm{h}$

\begin{tabular}{|c|c|c|c|c|c|c|}
\hline \multirow[b]{2}{*}{1} & Axle's weight (tons) & 7.9016 & 12.4905 & 5.0221 & 5.2576 & 5.4258 \\
\hline & Error (\%) & $8.29 \%$ & $-1.02 \%$ & $-8.83 \%$ & $-6.80 \%$ & $-7.15 \%$ \\
\hline \multirow{2}{*}{2} & Axle's weight (tons) & 7.7431 & 13.3150 & 4.6333 & 5.1742 & 5.2767 \\
\hline & Error (\%) & $6.12 \%$ & $5.52 \%$ & $-15.89 \%$ & $-8.28 \%$ & $-9.70 \%$ \\
\hline
\end{tabular}

Speed: $70 \mathrm{~km} / \mathrm{h}$

\begin{tabular}{|c|c|c|c|c|c|c|}
\hline \multirow{2}{*}{1} & Axle's weight (tons) & 7.8760 & 12.5578 & 5.1595 & 5.6120 & 5.6925 \\
\hline & Error (\%) & $7.95 \%$ & $-0.48 \%$ & $-6.34 \%$ & $-0.52 \%$ & $-2.59 \%$ \\
\hline \multirow{2}{*}{2} & Axle's weight (tons) & 7.6155 & 12.2707 & 5.0340 & 5.5636 & 5.6679 \\
\hline & Error (\%) & $4.37 \%$ & $-2.75 \%$ & $-8.62 \%$ & $-1.38 \%$ & $-3.01 \%$ \\
\hline \multicolumn{7}{|c|}{ Speed: 90 km/h } \\
\hline \multirow{2}{*}{1} & Axle's weight (tons) & 7.1292 & 13.3371 & 5.1899 & 5.7197 & 5.8324 \\
\hline & Error (\%) & $-2.29 \%$ & $5.69 \%$ & $-5.79 \%$ & $1.39 \%$ & $-0.19 \%$ \\
\hline \multirow{2}{*}{2} & Axle's weight (tons) & 7.6745 & 13.3600 & 5.1701 & 5.6840 & 5.8153 \\
\hline & Error (\%) & $5.18 \%$ & $5.87 \%$ & $-6.15 \%$ & $0.76 \%$ & $-0.48 \%$ \\
\hline
\end{tabular}

As it can be seen from the table (Table 3), most preferred for measurements are the velocity ranges from $70 \mathrm{~km} / \mathrm{h}$ and above, when the measurement errors of the load on the axle does not exceed $10 \%$, which is consistent with the problem of pre-selection of overloaded vehicles. This level of errors has the reason due to vertical oscillations of the dynamic motion of the vehicle, whose amplitudes are smaller at higher speeds. By the properties of each individual sensor, the calibration of FOS should be conducted twice: firstly, in the laboratory (load characteristics in the temperature range from $-20^{\circ} \mathrm{C}$ to $+30^{\circ} \mathrm{C}$ ), and, secondly, after installing the sensor in the road surface with vehicles with a standard load usage.

\section{Conclusions}

The model of seismic sensor excitation by automotive tyre footprint pressure is suggested. It is based on description of a footprint by certain discrete array considered as an equivalent source of a transient seismic signal. Proclaimed structure of the model is not contrary to widespread ideas of a motorcar wheel interaction with a road pavement. The model is displayed the temporal form of that pulse, which is described by a convolution matrix reflected the interference of Raleigh waves from different parts of the array. The appropriate computing procedures have been realized in Matlab. Resulted pulse forms may be used as a starting material for formulation of the target inverse problem in designing of WIM systems for estimation of loads on individual axles of a car.

The priori knowledge of the values and suspension stiffness of the tire, or one of them, is need for successful application of the method based on differential equations modelling. Unfortunately, the problem of determination of rigidity coefficient from the results of the optimisation has not been resolved, so we need to use its values from [14] or other sources now. Because of a weighted least square algorithm 
of global optimisation is used the processing times of WIM sensor signals are relatively long. It may be mentioned as some disadvantage of this approach. But this problem should be solved by parallel processing of the signals from each axle of the vehicle.

Dynamic WIM systems including FOS and reference database are not in using yet. The obtained results have to design that system for determination of the overloaded vehicles in traffic flows. The use of the reference systems, which are different in the structure, may increase the probability of the correct decision-making. One of the sounding problems is to create a set of the reference systems for different models of vehicles. The creation of such a set for the large number of vehicles is rather expensive venture. In this connection, the development of the automated systems that collects the statistical data and supports the reference identification systems of the overloaded vehicles is really actual problem.

To increase the accuracy of axes weight in the motion measurement it is necessary to select the area of data representation, where the static and dynamic components of the weight can be separated, let to be able to remove the dynamic components from the signal.

\section{Acknowledgements}

This research was granted by ERDF funding, project "Fibre Optic Sensor Applications for Automatic Measurement of the Weight of Vehicles in Motion: Research and Development (2010-2012)", No. 2010/0280/2DP/2.1.1.1.0/10/APIA/VIAA/094, 19.12.2010.

\section{References}

1. Jacob B., Feypell-de la Beaumelle, V. (2010). Improving truck safety: Potential of weigh-in-motion technology. IATSS Research, 34, 9-15.

2. Shengyao Jia et al. (2010). Signal acquisition and processing of the moving vehicle weighting system. WSEAS Trans. on Signal Proc., 3(6), 113-122.

3. Dorleus J. et al. (2009). A fibre optic seismic sensor for unattended ground sensing applications. ITEA Journ., 30, 455-460.

4. Mimbela, L. E. Y., Klein, L. A. (2005). Summary of vehicle detection and surveillance technologies used in intelligent transportation systems. New Mexico: New Mexico State University.

5. Mazurek, B. et al. (2001). Assessment of vehicle weight measurement method using PVDF transducers. J. of Electrostatics, 51-52, 76-81.

6. US Dept. of Commerce. (1997). Development of fibre optic dynamic WIM systems. Final Report. USA: Nat. Techn. Inf. Service.

7. Sheriff, R E., Geldart, L. P. (1985). Exploration seismology, V. 1. Cambridge: Cambridge Univ. Press.

8. Tatom, F. B., Herndon, G. W. (Feb. 2004). US Pat. No 6,692,567 B1. USA.

9. Tarasik, V. P. (2006). Theory of automobile motion. Saint-Petersburg: BHW. (In Russian)

10. Osinovskaya, V. A. (2006). About evaluation and prediction of automobile road vibrations. (In Russian), Retrieved 2006, from http://science-bsea.narod.ru/2006/story_2006/osinovskaya_vopros.htm

11. Mimbela, L.-E. Y., Pate, J., Copeland, S., Kent, P. M., Hamrick, J. (April 2003). Applications of Fibre Optic Sensors in Weigh-in-Motion (WIM) Systems for monitoring truck weights on pavements and structures. Final report on research project. New Mexico State University.

12. Levin, M. A., Fufayev, N. A. (1989). Theory of deformable rolling wheels. Moscow: Science. (In Russian)

13. Moazami, D., Muniandy, R., Hamid, H., Md.Yusoff, Z. (2011). Effect of tire footprint area in pavement response studies. International Journal of the Physical Sciences, 6(21), 5040-5047.

14. Balabin, I. V. (Ed.) (1985). Automotive and tractor wheels: Handbook. Moscow: Mashinostroenie. (In Russian)

15. Fernando, E. G., Musani, D., Dae-Wook, P., and Liu, W. (2006). Evaluation of Effects of Tire Size and Inflation Pressure on Tire Contact Stresses and Pavement Response. Project 0-4361. Texas, USA: Transportation Institute, College Station.

16. Smith, N. D. (2004). Understanding Parameters Influencing Tire Modelling. Colorado, USA: Colorado State University. Formula SAE Platform. 
17. Batenko, A., Grakovski, A., Kabashkin, I., Petersons, E., Sikerzhicki, Y. (2011). Weight-in-Motion (WIM) Measurements by Fiber Optic Sensor: Problems and Solutions. Transport and Telecommunication, 12(4), 27-33.

18. Peters, B., Koniditsiotis, C. (2000). Weigh-In-Motion Technology, Intermediate Report, CAustroads Inc., No. AP-R168/00. Burwood Highway, Australia: ARRB Transport Research Ltd.

19. Loo van, F. J. (2001). Project WIM-Hand, 1st interim report, DWW-Publication: IB-R-01-09, Road and Hydraulic Engineering Institute, DG Rijkswaterstaat.

20. Bushman, Rob, Pratt, Andrew J. (2002). Weigh In Motion Technology - Economics and Performance. In Proc. of NATMEC '02. Charlotte. North Carolina: Warren Publishing.

21. Giallorenzi, T. G., Bucaro, J. A., Dandridge, A., Siegel, G. II., Jr., Cole, J. II., Rashleighand, S. C., Priest, R. G. (1982). Optical Fiber Sensor Technology. IEEE Journal of Quantum Electronics, QE-18, pp. 626-665.

22. Scheuter, F. (1997). General Guide for Weighing with Portable Wheel Load Scales: HAENNI, Document P 1196. Maryland, Baltimore: Interscience Publishers.

23. Taylor, B., Klashinsky, R. (1995). New Application for Weigh-In-Motion Technology. Traffic Technology International. Surrey, England: UK \& International Press.

24. Sivuhin, D. (2005). General Physics Course. In Vol. I, Mechanics, $4^{\text {th }}$ Edition (560 p.). Moscow: FIZMALIT; MFTI Publ. (In Russian)

25. Widrow, B., Stearns, S. (1989). Adaptive Signal Processing. Moscow: Radio and Svjaz. (In Russian)

26. Shakun, P., Sikerzhicky, Y. (2012). Adaptive method's applications for the identification systems in dynamic weighing. In Proceedings of the 12th International Conference "Reliability and Statistics in Transportation and Communication" (RelStat'12), 17-20 October 2012 (pp. 296-306). Riga, Latvia: TTI.

27. Malla, Ramesh B., Sen, Amlan, Garrick, Norman W. (2008). A Special Fiber Optic Sensor for Measuring Wheel Loads of Vehicles on Highways. Sensors, 8, 2551-2568. Retrieved April 4, 2008, from MDPI database on the World Wide Web: http:// www.mdpi.org/sensors

28. SENSORLINE GmbH. (2010). SPT Short Feeder Spliceless Fiber Optic Traffic Sensor: product description. Sensor line, GmbH. Retrieved January 7, 2011, from http://sensorline.de/home/ pages/downloads.php

29. O’Brien, E. J., Jacob, B. (1998). European Specification on Vehicle Weigh-in-Motion of Road Vehicles. In Proceedings of the $2^{\text {nd }}$ European Conference on Weigh-in-Motion of Road Vehicles, 1998 (pp. 171-183). Luxembourg: Office for Official Publications of the European Communities.

30. Mesco, A. (1984) Digital Filtering: Applications in Geophysical Exploration for Oil, v. 1-2. Budapest: Academiai Kiado. 